\title{
Iterated Lower Bound Formulas: A Diagonalization-Based Approach to Proof Complexity*
}

\author{
Rahul Santhanam ${ }^{\dagger} \quad$ Iddo Tzameret ${ }^{\ddagger}$
}

September 23, 2021

\begin{abstract}
We propose a diagonalization-based approach to several important questions in proof complexity. We illustrate this approach in the context of the algebraic proof system IPS and in the context of propositional proof systems more generally.

We give an explicit sequence of CNF formulas $\left\{\phi_{n}\right\}$ such that VNP $\neq$ VP iff there are no polynomial-size IPS proofs for the formulas $\phi_{n}$. This provides the first natural equivalence between proof complexity lower bounds and standard algebraic complexity lower bounds. Our proof of this fact uses the implication from IPS lower bounds to algebraic complexity lower bounds due to Grochow and Pitassi together with a diagonalization argument: the formulas $\phi_{n}$ themselves assert the non-existence of short IPS proofs for formulas encoding VNP $\neq$ VP at a different input length. Our result also has meta-mathematical implications: it gives evidence for the difficulty of proving strong lower bounds for IPS within IPS.

More generally, for any strong enough propositional proof system $R$ we propose a new explicit hard candidate, the iterated $R$-lower bound formulas, which inductively asserts the non-existence of short $R$ proofs for formulas encoding this same statement at a different input length. We show that these formulas are unconditionally hard for Resolution following recent results of Atserias and Müller and of Garlik. We further give evidence in favour of this hypothesis for other proof systems.
\end{abstract}

\section{Introduction}

Diagonalization has been used to show many of the foundational results in logic, including Cantor's theorem about the uncountability of the reals, Gödel's Incompleteness Theorems, and Turing's proof of the undecidability of the Halting Problem. It has also found extensive application in complexity theory, where many unconditional lower bounds use diagonalization directly or indirectly. However, surprisingly in the area of resource bounded proofs, namely proof complexity, diagonalization has had very little impact so far.

Propositional proof complexity is concerned with the question of whether tautologies have short proofs in a given proof system. Cook and Reckhow [CR79] showed that NP $\neq$ coNP if and only if there are hard instances for every propositional proof system $R$, i.e., a sequence of instances that

\footnotetext{
*A preliminary version of this work appears in 53rd Ann. Symp. Theory of Comput., STOC 2021.

${ }^{\dagger}$ Department of Computer Science, Oxford University. rahul.santhanam@cs.ox.ac.uk. Supported in part by ERC Consolidator Grant Agreement No. 615075.

${ }^{\ddagger}$ Department of Computing, Imperial College London. iddo.tzameret@gmail.com. Part of this project has received funding from the European Research Council (ERC) under the European Union's Horizon 2020 research and innovation programme (grant agreement No. 101002742). Part of this work was done while on sabbatical visit to Oxford University, supported in part by ERC Consolidator Grant Agreement No. 615075.
} 
does not have short $R$-proofs. It is known that there are hard instances for relatively weak proof systems such as resolution [Hak85] and constant-depth Frege [Ajt88, KPW95, PBI93], but thus far it remains completely open whether the same is the case for strong proof systems like Frege and Extended Frege. Lower bounds on these systems seem extremely hard to attain, and there is even a shortage of good explicit candidates for hard instances.

In this paper, we propose a diagonalization-based approach to make progress on several important directions in proof complexity, including connections between proof complexity and circuit complexity, meta-mathematics of proof complexity lower bounds and explicit hard instances for propositional proof systems. We first give some background on each of these directions.

Connections Between Proof Complexity and Circuit Complexity: It is an intriguing fact that many of the known proof complexity lower bounds are shown using techniques that were originally developed in the context of circuit complexity, e.g., the technique of random restrictions. Intuitively, it seems as though progress on lower bounds in both areas is stalled for similar reasons, but there are few formal connections known between the two areas. For weak proof systems, the notion of feasible interpolation [Kra97] provides such a connection, enabling us to derive small circuits for certain computational problems from short proofs of related formulas. In the converse direction, recent lifting results [GKRS19] give a way to derive circuit complexity lower bounds for weak circuit classes from proof complexity lower bounds for related weak proof systems.

For strong proof systems, there are some intriguing connections known to algebraic complexity (cf. [PT16] for a survey). First, the strong algebraic proof system IPS (Ideal Proof System) introduced by Grochow and Pitassi in [GP18] was introduced in part to relate proof complexity lower bounds to complexity class separations such as VP vs. VNP. Furthermore, we know that some natural conjectures from algebraic circuit complexity, such as the Shub and Smale conjecture [SS95] about hardness of expressing factorial numbers with $0,1,-1$ constants and $\times,+$ gates, imply lower bounds on the IPS as shown recently in [AGHT20]. Moreover, unconditional lower bounds on some restricted versions of IPS have been shown to follow from certain algebraic circuits lower bounds in [FSTW16], while [LTW18] established connections between Frege lower bounds and lower bounds on the weak model of noncommutative formulas. Finding further connections is an important problem, so that progress in either area can be transferred to the other.

Meta-mathematics of Proof Complexity Lower Bounds: Proof complexity lower bounds seem to be difficult to show, but there seems to be little formal justification for this. In contrast, the difficulty of showing computational complexity lower bounds is evidenced by barriers such as the relativization barrier and the natural proofs barrier. Understanding the barriers to lower bounds better in the context of proof complexity might help us to make progress. One approach is via connections to circuit complexity. Grochow and Pitassi [GP18] show that super-polynomial lower bounds for CNFs in the algebraic proof system IPS imply VNP $\neq$ VP; thus if we believe VNP $\neq$ VP is hard to show, then we must believe the same for IPS lower bounds. In a recent work, [PS19] take a different approach, formulating an analogue of the natural proofs barrier for proof complexity. They show unconditionally that for some (non-uniform) propositional proof system $R$, super-polynomial lower bounds on $R$-proofs for random truth table formulas cannot be shown efficiently in any non-uniform propositional proof system. However, their proof is non-constructive, and does not seem to yield any new information for commonly studied proof systems such as Frege and Extended Frege.

Explicit Hard Instances for Propositional Proof Systems: Known proof complexity lower bounds for proof systems such as Resolution and constant-depth Frege hold for explicit 
formulas, indeed the Pigeonhole Principle is hard in both cases. However, for Frege and above, there are few explicit candidates for hard instances, as discussed in [Raz15]. Random CNFs of constant clause-to-variable density and random truth table formulas are plausible candidates, but are not explicit. The only plausible explicit candidates of which we are aware, apart from canonical examples such as reflection principles for strong proof systems ${ }^{1}$, are explicit circuit lower bound tautologies and the more general proof complexity generators [ABSRW04, Kra01]. However, even explicit circuit lower bound tautologies are no longer hard candidates for strong propositional proof systems that can prove circuit lower bounds - such a strong proof system can be defined by adding a circuit lower bound tautology as an axiom to Frege, if circuit lower bounds do indeed hold for functions in exponential time. In general, there is a need for more plausible candidates for hardness, prior to any approach to showing lower bounds for strong proof systems.

We make progress along the three directions above in the context of the algebraic proof system IPS and for propositional proof systems in general. We next explain our results.

\subsection{Our Results}

In the first part of this work we show how our diagonlisation applies to strong algebraic proof systems with relation to algebraic circuit lower bounds. In the second part we introduce our iterated lower bounds formulas as a means to attain proof complexity lower bounds via diagonalisation for general propositional proof systems.

\subsubsection{Proof complexity characterisation of $V P \neq V N P$}

Our main technical result is an equivalence between super-polynomial algebraic circuit lower bounds for the Permanent and non-existence of IPS short refutations for a certain sequence of explicit CNFs. In the following informal statement of the result we use "VNP $\neq$ VP" to denote appropriate CNF encodings of VNP $\neq$ VP (itself a sequence of statements asserting that the Permanent, as given by its list of monomials at a given input length, lacks small algebraic circuits) over a finite field $\mathbb{F}$, and $\mathrm{Ib}_{I P S}\left(\right.$ " $\phi_{n}$ ", $\left.s\right)$ to denote appropriate CNF encodings of the statements that there are no IPS-proofs of $\phi_{n}$ of size $s(|\phi|)$, where $s: \mathbb{N} \rightarrow \mathbb{N}$ is a function (below, $m$ refers to the size of "VNP $\neq \mathrm{VP}$ "). ${ }^{2}$

Theorem 1.1 (Equivalence between Algebraic Circuit Lower Bounds and IPS Lower Bounds; informal). VNP $\neq$ VP over finite fields iff there is a constant $c$ such that there are no polynomialsize IPS proofs of the CNF formula $\mathrm{Ib}_{I P S}$ ("VNP $\left.\neq \mathrm{VP} ", m^{c}\right)$.

Note that the explicit CNF formulas which lack efficient IPS proofs iff VNP $\neq$ VP are themselves encodings of IPS lower bounds for the statement VNP $\neq$ VP - this is how we use diagonalization. Our argument combines diagonalization ideas with the result of Grochow and Pitassi [GP18] that IPS lower bounds for CNFs imply circuit lower bounds. We give more details on the proof ideas in the next section. Our proof ideas generalize to give an analogue of Thm. 1.1 for every algebraic proof system that efficiently simulates IPS.

It is important to clarify that the formulas $\mathrm{Ib}_{I P S}$ ("VNP $\neq \mathrm{VP} ", m^{c}$ ) are not necessarily tautologies: we do not know if there are IPS lower bounds against proving VNP $\neq$ VP (let alone whether VNP $\neq$ VP holds). We work with formulas that are conjectured to be tautologies, and show that for these conjectured tautologies, their proof complexity hardness is equivalent to VNP $\neq \mathrm{VP}$. It is possible that VNP $\neq$ VP actually has polynomial-size IPS proofs, in which case Thm. 1.1 holds

\footnotetext{
${ }^{1}$ That is, principles expressing the soundness of proof systems that are conjectured to be stronger than the proof system we wish to lower bound.

${ }^{2}$ Here and elsewhere we abuse the terminology by writing "IPS proof of a CNF formula expressing a statement" to formally mean an IPS refutation refuting the unsatisfiable CNF expressing the negation of the statement.
} 
because $\mathrm{Ib}_{I P S}$ ("VNP $\neq \mathrm{VP}$ ", $m^{c}$ ) is not a tautology and hence trivially lacks small IPS proofs. This, however, cannot happen if we assume a natural algebraic analogue of Razborov's Conjecture [Raz15, Kra04b] as we now explain.

Razborov conjectured that under standard circuit complexity assumptions, Frege cannot efficiently prove super-polynomial circuit lower bounds for any Boolean function (as expressed in the so-called truth table formulas). Let us formulate a reasonable algebraic analogue of Razborov's Conjecture: IPS cannot efficiently prove super-polynomial algebraic circuit lower bounds for any polynomial. Note that this algebraic variant of Razborov's conjecture, ruling out short IPS proofs of VNP $\neq \mathrm{VP}$, does not rule out short proofs of the statement expressing that "there are no short $I P S$ proofs of VNP $\neq$ VP". Thm. 1.1 shows that assuming this variant of Razborov's conjecture, $\mathrm{VNP} \neq \mathrm{VP}$ is equivalent to a lower bound on IPS proofs of this latter statement. ${ }^{3}$

Thm. 1.1 is relevant to two of the three directions we cited as motivation earlier. It gives a new connection between algebraic complexity and proof complexity, which could be useful in either direction. The proof of Thm. 1.1 also provides an argument that can be applied more generally: it shows a way to flip the direction of any reduction that reduces proof hardness to circuit hardness, to obtain a reduction that reduces circuit hardness to proof hardness (or more precisely to non-efficient provability), as explained in Subsection 1.2. Thm. 1.1 is also relevant to the meta-mathematics of IPS lower bounds. For a certain natural explicit family of formulas expressing algebraic circuit lower bounds for the Permanent, super-polynomial IPS lower bounds are themselves hard to show in IPS, if we believe that VNP $\neq$ VP. Thus, under reasonable complexity conjectures, namely that VNP $\neq$ VP and that the algebraic analogue of Razborov's conjecture holds, IPS finds it hard to reason about itself.

\subsubsection{Iterated lower bounds formulas}

Thm. 1.1 gives evidence that IPS finds it hard to reason efficiently about itself - could this hold for proof systems more generally ${ }^{4}$ ? A heuristic way to think of Thm. 1.1 is as a fixed point result in the following sense: consider $\mathrm{Ib}_{R}\left(\cdot, m^{\omega(1)}\right)$ for a proof system $R$ as an operator mapping formulas to formulas, namely $\varphi \mapsto \mathrm{lb}_{R}\left(\varphi, m^{\omega(1)}\right)$ (in applications we take instead of $m^{\omega(1)}$ a concrete polynomial lower bound $m^{c}$, for a constant $c$ ). Let $\mathrm{lb}_{R}^{2}$ be the composition of this operator with itself, namely $\varphi \mapsto \mathrm{Ib}_{R}\left(\mathrm{Ib}_{R}\left(\varphi, m^{\omega(1)}\right), m^{\omega(1)}\right)$. Then the sequence of formulas expressing that VNP $\neq \mathrm{VP}$ is a fixed point for $\mathrm{Ib}_{R}^{2}$ in the sense that it preserves truth when $R$ is IPS: if VNP $\neq$ VP holds then $\mathrm{Ib}_{R}\left(\mathrm{Ib}_{R}\left(\mathrm{VNP} \neq \mathrm{VP}, m^{\omega(1)}\right), m^{\omega(1)}\right)$ holds. Indeed, our diagonalisation approach is inspired partly by Atserias and Müller, and Garlik [AM20, Gar19], who showed implicitly that every sequence of formulas is such a fixed point for $\mathrm{Ib}_{R}^{2}$ when $R$ is Resolution, and by [PS19] who showed implicitly that for every strong enough (nonuniform) propositional proof system $R$ (simulating Extended Frege), the distribution of random truth table formulas is a fixed point for $\mathrm{Ib}_{R}^{2}$.

Here we explore the idea that iterating $\mathrm{lb}_{R}$ provides an explicit hard sequence of formulas for $R$. Assume that $R$ is not polynomially bounded, and let $\phi$ be a fixed formula that does not have $|\phi|^{c}$ size $R$-proofs, for some constant $c$.

\footnotetext{
${ }^{3}$ Razborov conjectured in [Raz15] that Frege cannot efficiently prove super-polynomial circuit lower bounds for any Boolean function. More specifically, [Raz15, Conjecture 1] with suitable parameters for the underlying combinatorial designs implies under some hardness assumptions that Frege cannot efficiently prove that SAT $\nsubseteq$ P/poly. Further conjectures about the impossibility of Extended Frege to efficiently prove circuit lower bounds have been circulated in the proof complexity literature and discussions (cf. [Raz16, Raz21, Kra11]).

${ }^{4}$ Independently of our work, Pudlák [Pud20] poses the same question.
} 
We define the iterated lower bound formulas $\mathrm{Ib}_{R}^{k}\left(\phi, n^{c}\right)$ inductively as follows:

1. $\operatorname{lb}_{R}^{0}\left(\phi, n^{c}\right)=\phi$;

2. $\mathrm{Ib}_{R}^{k+1}\left(\phi, n^{c}\right)=\mathrm{Ib}_{R}\left(\mathrm{Ib}_{R}^{k}\left(\phi, n^{c}\right), n^{c}\right)$.

We propose the Iterated Lower Bound Hypothesis: for every reasonably strong ${ }^{5}$ propositional proof system $R$ that is not polynomially bounded, there is a $\phi$ such that the sequence $\left\{\mathrm{lb}_{R}^{k}\left(\phi, n^{c}\right)\right\}_{k=0}^{\infty}$ is a sequence of hard instances for $R$.

This generically gives a candidate family of hard instances for every strong enough proof system. We believe that there is a win-win aspect to studying this hypothesis - even if it fails, this gives us information about whether propositional proof systems can reason about lower bounds for themselves. We are not currently aware of any natural propositional proof system $R$ that is capable of this. Though there have been discussions on whether constant-depth Frege for example can efficiently prove its known lower bounds, even if this is the case our hypothesis would make sense for strong proof systems, as well as for weak proof systems.

We provide evidence in favour of the hypothesis. First, as a corollary to [AM20, Gar19], it follows that the hypothesis holds for Resolution.

Theorem 1.2. The Iterated Lower Bounds Hypothesis holds for Resolution.

Second, we show that under a conjecture of Rudich [Rud97] about non-existence of short propositional proofs for random truth table formulas, any finite number of iterations preserves hardness for random truth table formulas, for some strong propositional proof system $R$ that efficiently simulates Extended Frege.

Theorem 1.3. Assuming Rudich's Conjecture, there is a propositional proof system $R$ efficiently simulating Extended Frege such that for every large enough constant $c$ and every fixed positive integer $k, \mathrm{~b}_{R}^{k}\left(\phi,|\phi|^{c}\right)$ does not have $R$-proofs of size $\left|\mathrm{Ib}_{R}^{k}\left(\phi,|\phi|^{c}\right)\right|^{c}$ with high probability over the truth table formula $\phi$ expressing that a random Boolean function on $n$ variables does not have circuits of size $n^{c}$.

\subsection{Overview of Techniques}

We give a high-level overview of the ideas required to show Thm. 1.1. Informally, we would like to show that VNP $\neq$ VP iff IPS cannot efficiently prove IPS lower bounds for "VNP $\neq$ VP".

The proof builds on several technical constructions. The gist of the argument is a form of diagonalisation: the existence of a short proof of a proof complexity lower bound for the statement VNP $\neq$ VP implies in fact that there $i s$ a short proof of VNP $\neq$ VP (at a smaller input length) due to the reduction of Grochow and Pitassi [GP18], that we show is efficiently formalizable already inside IPS. Note that as we mentioned above in Sect.1.1.1, this argument can be applied more generally: for any proof system $P$ and any circuit lower bound statement denoted Circuit-Hard, the existence of a short $P$ proof of the statement that $P$ does not admit short proofs of Circuit-Hard implies in fact that there is a short $P$ proof of Circuit-Hard (at a smaller input length), assuming a proof complexity lower bound on $P$ proofs implies Circuit-Hard (and as long as we can formalise this implication efficiently in $P$ ).

For the purpose of our argument we make the following assumptions, which we will justify later: (1) There is a reasonable CNF encoding of "VNP $\neq$ VP"; (2) there is a reasonable CNF encoding of the statement that there are no IPS lower bounds of size $s$ for a CNF $\phi ;(3)$ if $\phi$ is a tautology and

\footnotetext{
${ }^{5}$ We formally define "reasonably strong" later.
} 
there are short IPS proofs of "IPS does not efficiently prove $\phi$ ", then there are short IPS proofs of "VNP $\neq$ VP". Assumption 3 can be thought of as the formalization of the Grochow-Pitassi implication from IPS lower bounds for CNFs to VNP $\neq$ VP within IPS. A priori, it is hard to see how to establish Assumption 3 since we only know that $\phi$ is a tautology and do not have proofs of this fact. It will turn out that the parameters can be set so that truth-table proofs of $\phi$ suffice.

Given these assumptions, we proceed as follows. First, we show the forward direction. Assume VNP $\neq$ VP. Then "VNP $\neq$ VP" is a CNF tautology, using Assumption 1. Assume for the sake of contradiction that $\mathrm{Ib}_{I P S}$ ("VNP $\neq \mathrm{VP}$ ", $n^{\omega(1)}$ ) has polynomial-size proofs in IPS. Then by Assumption 3 , "VNP $\neq$ VP" has polynomial-size proofs in IPS. But this contradicts the soundness of IPS, since IPS has polynomial-size proofs of the statement that "VNP $\neq$ VP" requires superpolynomial-size IPS-proofs.

For the backward direction, either $\phi=" \mathrm{~b}_{I P S}$ ("VNP $\left.\neq \mathrm{VP} ", n^{\omega(1)}\right)$ " is true or it is not. If it is true, then $\mathrm{Ib}_{I P S}\left(\phi, n^{\omega(1)}\right)$ implies VNP $\neq \mathrm{VP}$ by [GP18], using Assumption 2. If it is false, then "VNP $\neq$ VP" has poly-size proofs in IPS. By the soundness of IPS, this implies VNP $\neq$ VP.

The above is only a template and hides many technical issues and details. For example, in practice, we work with the statement VNP $\neq$ VP at a given input length, and we need to clarify how the input lengths of different occurrences of the statement relate to each other.

Technically, for the sake of the formalisation in IPS we need to be able to speak at the IPS proof level about circuit lower bounds, proof complexity lower bounds, and the reduction between them as in [GP18]. To formalise statements about IPS proofs and circuit class separations we express polynomials as vectors of coefficients. To express the existence of small circuits we use universal circuits as defined in Raz [Raz10]. To express that a given polynomial is computable by a small circuit we use a set of equations. Each equation states that the coefficient of a monomial computed by the universal circuit has the appropriate value.

Using this formalisation of polynomials computed by small circuits we can encode VP $\neq$ VNP as the statement expressing that the coefficient vector of the permanent polynomial is not equal to the coefficient vector of any small universal circuit. Similarly, the IPS proof predicate is expressed by stating the existence of a small universal circuit that computes (similarly, based on its monomial coefficients) the IPS certificate of a given CNF. For the purpose of expressing statements about algebraic circuits such as VP $\neq$ VNP as CNF formulas we first need to work over finite fields, and second need to devise ways to move from CNF formulas encoding circuits to the circuit they express.

\subsection{Related Work}

Following on unpublished work of Friedman [Fri79], Pudlak [Pud86, Pud87] showed finitistic versions of Gödel's Theorem: for any strong enough first-order theory $T$ of arithmetic, there is a constant $\epsilon>0$ such that the finitistic consistency principle $\operatorname{Con}_{T}(n)$ stating that there are no $T$-proofs of $1=0$ of size at most $n$ requires T-proofs of size at least $n^{\epsilon}$. For first-order theories satisfying additional properties, stronger lower bounds approaching $n$ can be obtained [Pud87]. By the standard translation between first-order theories and propositional proof systems, this yields non-trivial lower bounds on the $Q$-proof size of the reflection principle for $Q$ when $Q$ is a strong enough propositional proof system. However, the lower bounds obtained in this way are sub-linear in the formula size, while we are interested in the question of super-polynomial size lower bounds. Note that the reflection principle in fact has proofs of polynomial size [Pud86].

Diagonalization techniques have also been explored in work of Krajicek [Kra04c, Kra04a]. In [Kra04c], the notion of implicit proofs is defined and studied. This concept is used in [Kra04a] to show a conditional result: if $E$ requires exponential-size circuits and NP $=$ coNP, then there is no 
p-optimal propositional proof system. Our techniques here are very different, and our results seem unrelated to those in [Kra04a].

On the other hand, the results of Grochow and Pitassi [GP18] are crucial to our work. Grochow and Pitassi defined the Ideal Proof System, an algebraic proof system for which proofs are verifiable by polynomial size circuits (and in polynomial time under a standard derandomization assumption). They showed that super-polynomial IPS lower bounds for CNFs would imply VNP $\neq$ VP. This connection between proof complexity lower bounds and circuit complexity lower bounds has been further developed in [FSTW16, LTW18, AGHT20]. However, none of these previous works establish an equivalence between proof complexity lower bounds and standard circuit lower bounds, as in Theorem Thm. 1.1.

We are also inspired by recent work of [AM20, PS19]. Atserias and Muller [AM20] settle the long-standing open problem of whether Resolution is automatable (assuming $P \neq N P$ ) by giving a reduction from SAT to proof complexity lower bounds for Resolution via variants of the proof complexity lower bound formulas. Their reduction relies on the hardness of the Pigeonhole Principle, which only holds for weak proof systems such as Resolution, while we are interested here in strong proof systems such as IPS. Pich and Santhanam [PS19] unconditionally establish a version of the Natural Proofs barrier [RR97] in proof complexity, but they do so for a proof system that is defined non-constructively and moreover for instances which are randomly generated. In contrast, we are interested here in the meta-mathematics of proof complexity for well-studied and concrete proof systems such as IPS and for explicit instances. We do use ideas from [AM20, PS19] to give evidence for the Iterated Lower Bounds Hypothesis.

Finally, the idea of iterating proof complexity lower bounds is novel to the best of our knowledge. Nevertheless, as discussed above, considering the proof complexity of proof complexity lower bounds has been investigated to different degrees of explicitness in the literature. And we refer the reader to the recent excellent survey by Pudlak [Pud20] that explores this and other related questions.

\section{Preliminaries}

\subsection{Basic Algebraic Complexity}

For an excellent treatise on algebraic circuits and their complexity see Shpilka and Yehudayoff [SY10]. Let $\mathbb{G}$ be a ring. Denote by $\mathbb{G}[X]$ the ring of (commutative) polynomials with coefficients from $\mathbb{G}$ and variables $X:=\left\{x_{1}, x_{2}, \ldots\right\}$. A polynomial is a formal linear combination of monomials, where a monomial is a product of variables. Two polynomials are identical if all their monomials have the same coefficients. The degree of a polynomial is the maximal total degree of a monomial in it.

Algebraic circuits and formulas over the ring $\mathbb{G}$ compute polynomials in $\mathbb{G}[X]$ via addition and multiplication gates, starting from the input variables and constants from the ring. More precisely, an algebraic circuit $C$ is a finite directed acyclic graph (DAG) with input nodes (i.e., nodes of indegree zero) and a single output node (i.e., a node of out-degree zero). Input nodes are labeled with either a variable or a ring element in $\mathbb{G}$. All the other nodes have fan-in (that is, in-degree) two and are labeled by either an addition gate + or a product gate $\times$. Every node in an algebraic circuit $C$ computes a polynomial as follows: an input node computes the variable or scalar that labels it. A $+($ or $\times)$ gate is said to compute the addition (product, resp.) of the (commutative) polynomials computed by its incoming nodes. The polynomial computed by a node $u$ in an algebraic circuit $C$ is denoted $\widehat{u}$. Given a circuit $C$, we denote by $\widehat{C}$ the polynomial computed by $C$, that is, the polynomial computed by the output node of $C$. The size of a circuit $C$ is the number of nodes in it, denoted $|C|$, and the depth of a circuit is the length of the longest directed path in it. For an algebraic circuit $C$ we write $C(a / x)$ to denote the substitution instance of $C$ in which every 
occurrence of the node $x$ is replaced by the sub-circuit $a$; in case $C(x)$ is written with its displayed variable(s) $x$ we can write $C(x)(a / x)$ for this substitution instance. We say that a polynomial is homogeneous whenever every monomial in it has the same (total) degree.

Definition 2.1 (Syntactic-degree $\operatorname{sdeg}(\cdot))$. Let $C$ be a circuit (without division) and $v$ a node in $C$. The syntactic-degree $\operatorname{sdeg}(\mathrm{v})$ of $v$ is defined as follows:

1. If $v$ is a field element or a variable, then $\operatorname{sdeg}(\mathrm{v}):=0$ and $\operatorname{sdeg}(\mathrm{v}):=1$, respectively;

2. If $v=u+w$ then $\operatorname{sdeg}(\mathrm{v}):=\max \{\operatorname{sdeg}(\mathrm{u}), \operatorname{sdeg}(\mathrm{w})\}$;

3. If $v=u \cdot w$ then $\operatorname{sdeg}(\mathrm{v}):=\operatorname{sdeg}(\mathrm{u})+\operatorname{sdeg}(\mathrm{w})$.

An algebraic circuit is said to be syntactic-homogeneous if for every plus gate $u+v, \operatorname{deg}(u)=$ $\operatorname{deg}(v)$.

Algebraic Complexity Classes. We now recall some basic notions from algebraic complexity (for more details see [SY10, Sec. 1.2]). Over a ring $R, \mathrm{VP}_{R}$ (for "Valiant's P") is the class of families $f=\left(f_{n}\right)_{n=1}^{\infty}$ of formal polynomials $f_{n}$ such that $f_{n}$ has poly $(n)$ input variables, is of poly $(n)$ degree, and can be computed by algebraic circuits over $R$ of poly $(n)$ size. VNP $R$ (for "Valiant's NP") is the class of families $g$ of polynomials $\left(g_{n}\right)_{n=1}^{\infty}$ such that $g_{n}$ has poly $(n)$ input variables and is of poly $(n)$ degree, and can be written as

$$
g_{n}\left(x_{1}, \ldots, x_{\operatorname{poly}(n)}\right)=\sum_{\bar{e} \in\{0,1\} \operatorname{poly}(n)} f_{n}(\bar{e}, \bar{x})
$$

for some family $\left(f_{n}\right)_{n=1}^{\infty} \in \mathrm{VP}_{R}$.

A polynomial $f(\bar{x})$ is a projection of a polynomial $g(\bar{y})$ if $f(\bar{x})=g(L(\bar{x}))$ identically as polynomials in $\bar{x}$, for some map $L$ that assigns to each $y_{i}$ either a variable or a constant. In other words, a projection of $g(\bar{y})$ is a substitution instance of $g(\bar{y})$ in which $\bar{y}$ variables are substituted by $\bar{x}$ variables or field elements. A family of polynomials $\left(f_{n}\right)$ is a polynomial projection or p-projection oft another family $\left(g_{n}\right)$ if there is a function $t(n)=n^{\Theta(1)}$ such that $f_{n}$ is a projection of $g_{t(n)}$ for all (sufficiently large) $n$. The permanent polynomial $\sum_{\sigma \in S_{n}} \prod_{i=1}^{n} x_{i, \sigma(i)}$ (for $S_{n}$ the permutation group on $n$ elements) is complete under p-projections for VNP when the ring $R$ is a field of characteristic different from 2. The determinant polynomial on the other hand is known to be in VP but is not known to be complete for VP under p-projections.

Two central questions in algebraic complexity theory are whether the permanent is a pprojection of the determinant (a stronger variant speaks about quasi-polynomial projections); and whether VP equals VNP [Val79b, Val79a, Val82]. Since the permanent is complete for VNP (under p-projections), showing $\mathrm{VP} \neq \mathrm{VNP}$ amounts to proving that the permanent cannot be computed by polynomial-size algebraic circuits.

\subsection{Algebraic Proof Systems}

Grochow and Pitassi [GP18] suggested the following algebraic proof system which is essentially a Nullstellensatz proof system $\left(\left[\mathrm{BIK}^{+} 96\right]\right)$ written as an algebraic circuit. A proof in the Ideal Proof System is given as a single polynomial. We provide below the boolean version of IPS (which includes the boolean axioms), namely the version that establishes the unsatisfiability over 0-1 of a set of polynomial equations. In what follows we follow the notation in [FSTW16]: 
Definition 2.2 ((boolean) Ideal Proof System (IPS), Grochow-Pitassi [GP18]). Let $f_{1}(\bar{x}), \ldots, f_{m}(\bar{x}), p(\bar{x})$ be a collection of polynomials in $\mathbb{F}\left[x_{1}, \ldots, x_{n}\right]$ over the field $\mathbb{F}$. An IPS proof of $p(\bar{x})=0$ from $\left\{f_{j}(\bar{x})=0\right\}_{j=1}^{m}$, showing that $p(\bar{x})=0$ is semantically implied from the assumptions $\left\{f_{j}(\bar{x})=0\right\}_{j=1}^{m}$ over 0-1 assignments, is an algebraic circuit $C(\bar{x}, \bar{y}, \bar{z}) \in$ $\mathbb{F}\left[\bar{x}, y_{1}, \ldots, y_{m}, z_{1}, \ldots, z_{n}\right]$ such that (the equalities in what follows stand for formal polynomial identities $\left.^{6}\right)$ :

1. $C(\bar{x}, \overline{0}, \overline{0})=0$; and

2. $C\left(\bar{x}, f_{1}(\bar{x}), \ldots, f_{m}(\bar{x}), x_{1}^{2}-x_{1}, \ldots, x_{n}^{2}-x_{n}\right)=p(\bar{x})$.

The size of the IPS proof is the size of the circuit $C$. The variables $\bar{y}, \bar{z}$ are called the placeholder variables since they are used as placeholders for the axioms. An IPS proof $C(\bar{x}, \bar{y}, \bar{z})$ of $1=0$ from $\left\{f_{j}(\bar{x})=0\right\}_{j \in[m]}$ is called an IPS refutation of $\left\{f_{j}(\bar{x})=0\right\}_{j \in[m]}$ (note that in this case it must hold that $\left\{f_{j}(\bar{x})=0\right\}_{j=1}^{m}$ have no common solutions in $\left.\{0,1\}^{n}\right)$.

Notice that the definition above adds the equations $\left\{x_{i}^{2}-x_{i}=0\right\}_{i=1}^{n}$, called the set of boolean axioms denoted $\bar{x}^{2}-\bar{x}$, to the system $\left\{f_{j}(\bar{x})=0\right\}_{j=1}^{m}$. This allows to refute over $\{0,1\}^{n}$ unsatisfiable systems of equations. Also, note that the first equality in the definition of IPS means that the polynomial computed by $C$ is in the ideal generated by $\bar{y}, \bar{z}$, which in turn, following the second equality, means that $C$ witnesses the fact that 1 is in the ideal generated by $f_{1}(\bar{x}), \ldots, f_{m}(\bar{x}), x_{1}^{2}-$ $x_{1}, \ldots, x_{n}^{2}-x_{n}$ (the existence of this witness, for unsatisfiable set of polynomials, stems from the Nullstellensatz theorem $\left.\left[\mathrm{BIK}^{+} 96\right]\right)$.

In order to use IPS as a propositional proof system (namely, a proof system for propositional tautologies), we need to fix the encoding of clauses as algebraic circuits.

Definition 2.3 (algebraic translation of CNF formulas). Given a CNF formula in the variables $\bar{x}$, every clause $\bigvee_{i \in P} x_{i} \vee \bigvee_{j \in N} \neg x_{j}$ is translated into $\prod_{i \in P}\left(1-x_{i}\right) \cdot \prod_{j \in N} x_{j}=0$. (Note that these terms are written as algebraic circuits as displayed, where products are not multiplied out.)

Notice that in this way a 0-1 assignment to a CNF is satisfying iff the assignment is satisfying all the equations in the algebraic translation of the CNF.

Therefore, using Definition 2.3 to encode CNF formulas, boolean IPS is considered as a propositional proof system for the language of unsatisfiable CNF formulas, sometimes called propositional $I P S$. We say that an IPS proof is an algebraic IPS proof, denoted IPS ${ }^{\text {alg }}$, if we do not use the boolean axioms $\bar{x}^{2}-\bar{x}$ in the proof. In our applications we are going to use algebraic IPS refutations, while sometimes explicitly adding the boolean axioms for some variables (while leaving them out for some other variables). As a default when referring to IPS we mean the boolean IPS version. When we use algebraic IPS we will say that explicitly.

The following is the main structural-complexity result for IPS. Notice that it already works for algebraic IPS and this will be important for us.

Theorem 2.4 (Grochow-Pitassi [GP18]). For any ring R, a super-polynomial lower bound on algebraic IPS refutations (and hence also on IPS refutations) over $R$ for any family of CNF formulas implies $\mathrm{VNP}_{R} \neq \mathrm{VP}_{R}$. The same result hold if we assume that the IPS refutation size lower bound holds only infinitely often.

The following lemma is the key to the proof of the Theorem, and is used in our application:

Lemma 2.5. Every family of unsatisfiable CNF formulas $\left(\varphi_{n}\right)$ has a family of algebraic IPS (and hence also of IPS) certificates $\left(C_{n}\right)$ in $\mathrm{VNP}_{R}$.

\footnotetext{
${ }^{6}$ That is, $C(\bar{x}, \overline{0}, \overline{0})$ computes the zero polynomial and $C\left(\bar{x}, f_{1}(\bar{x}), \ldots, f_{m}(\bar{x}), x_{1}^{2}-x_{1}, \ldots, x_{n}^{2}-x_{n}\right)$ computes the polynomial $p(\bar{x})$.
} 
Proof of Thm. 2.4, assuming Lemma 2.5. For a given set $\mathcal{F}$ of unsatisfiable polynomial equations $F_{1}=\cdots=F_{m}=0$, a lower bound on algebraic IPS refutations of $\mathcal{F}$ is equivalent to giving the same circuit lower bound on all IPS certificates for $\mathcal{F}$. A super-polynomial lower bound on IPS implies that some function in VNP - namely, the VNP-IPS certificate guaranteed by Lemma 2.5 - cannot be computed by polynomial-size algebraic circuits, and hence that VNP $\neq$ VP.

\subsubsection{Conventions and Notations for IPS Proofs}

An IPS (algebraic or not) proof over a specific field or ring is sometimes denoted IPS $\mathrm{S}_{\mathbb{F}}$ specifying explicitly it is over $\mathbb{F}$. For two algebraic circuits $F, G$, we define the size of the circuit equation $F=G$ to be the total circuit size of $F$ and $G$, namely, $|F|+|G|$. For a set $\overline{\mathcal{F}}$ of equations between circuits we denote by $|\overline{\mathcal{F}}|$ to be the total size of the equations in the set.

Let $\overline{\mathcal{F}}$ denote a set of polynomial equations $\left\{f_{i}(\bar{x})=0\right\}_{i=1}^{m}$, and let $C(\bar{x}, \bar{y}, \bar{z}) \in \mathbb{F}[\bar{x}, \bar{y}, \bar{z}]$ be an IPS proof of $f(\bar{x})$ from $\overline{\mathcal{F}}$ as in Definition 2.2. Then we write $C\left(\bar{x}, \overline{\mathcal{F}}, \bar{x}^{2}-\bar{x}\right)$ to denote the circuit $C$ in which $y_{i}$ is substituted by $f_{i}(\bar{x})$ and $z_{i}$ is substituted by the boolean axiom $x_{i}^{2}-x_{i}$. By a slight abuse of notation we also call $C\left(\bar{x}, \overline{\mathcal{F}}, \bar{x}^{2}-\bar{x}\right)=f(\bar{x})$ an IPS proof of $f(\bar{x})$ from $\overline{\mathcal{F}}$ and $\bar{x}^{2}-\bar{x}$ (that is, displaying $C(\bar{x}, \bar{y}, \bar{z})$ after the substitution of the placeholder variables $\bar{y}, \bar{z}$ by the axioms in $\overline{\mathcal{F}}$ and $\bar{x}^{2}-\bar{x}$, respectively).

The following fact shows that polynomial identities are proved for free in IPS:

Fact 2.6 ([AGHT20]). If $F(\bar{x})$ is a circuit in the variables $\bar{x}$ over the field $\mathbb{F}$ that computes the zero polynomial, then there is an IPS proof of $F(\bar{x})=0$ of size $|F|$.

Proof of fact. The IPS proof of $F(\bar{x})=0$ is simply $C(\bar{x}, \bar{z}):=F(\bar{x})$ (note that we do not need to use the boolean axioms nor any other axioms in this case). Observe that both conditions 1 and 2 for IPS hold in this case (Definition 2.2).

For two polynomials $f(\bar{x}), g(\bar{x})$, an IPS proof of $f(\bar{x})=g(\bar{x})$ from the assumptions $\overline{\mathcal{F}}$ is an IPS proof of $f(\bar{x})-g(\bar{x})=0$ (note that in case $f(\bar{x})$ and $g(\bar{x})$ are identical as polynomials this is trivial to prove by Fact 2.6).

We denote by $C: \overline{\mathcal{F}} \frac{s}{\operatorname{IPS}} p=0$ (resp. $C: \overline{\mathcal{F}} \mid \frac{s}{\operatorname{IPS}} p=g$ ) the fact that $p=0$ (resp. $p=g$ ) has an IPS proof $C(\bar{x}, \bar{y}, \bar{z})$ of size $s$ from assumptions $\overline{\mathcal{F}}$, and we use $\frac{s, d}{\operatorname{IPS}}$ when we want to specify that the degree of the IPS proof is upper bounded by $d$. Assumptions $\overline{\mathcal{F}}$ can be written either as a set of equations or as a sequence of equations or sets thereof separated by commas. We may also suppress "=0" and write simply $C: \overline{\mathcal{F}} \mid \frac{s}{\operatorname{IPS}} p$ for $C: \overline{\mathcal{F}} \mid \frac{s}{\operatorname{IPS}} p=0$. Whenever we are only interested in claiming the existence of an IPS proof of size $s$ of $p=0$ from $\overline{\mathcal{F}}$ we suppress the $C$ from the notation. Similarly, we can suppress the size parameter $s$ from the notation. If $F$ is a circuit computing a polynomial $\widehat{F} \in \mathbb{F}[\bar{x}]$, then we can talk about an IPS proof $C$ of $F$ from assumptions $\overline{\mathcal{F}}$, in symbols $C:\left.\overline{\mathcal{F}}\right|_{\text {IPS }} F$, meaning an IPS proof of $\widehat{F}$. Accordingly, for two circuits $F$, $F^{\prime}$ such that $\widehat{F}=\widehat{F}^{\prime}$, we may speak about an an IPS proof $C$ of $F$ from assumptions $\overline{\mathcal{F}}$ to refer to an IPS proof of $F^{\prime}$ from assumptions $\overline{\mathcal{F}}$. If $\left\{p_{i}=0\right\}_{i=1}^{\infty}$ and $\left\{\overline{\mathcal{F}}_{i}\right\}_{i=0}^{\infty}$ are sequences of circuit equations and sets of circuit equations, respectively, then we write $\overline{\mathcal{F}}_{i} \underset{\text { IPS }}{\stackrel{*}{\text { IS }}} p_{i}=0$ to denote that there is an IPS proof of $p_{i}=0$ from the assumptions $\overline{\mathcal{F}}_{i}$ of size polynomial in $\left|\overline{\mathcal{F}}_{i}\right|+\left|p_{i}\right|$.

When we deal with algebraic IPS proofs we will use the same notation as above, only using IPS $^{\text {alg }}$ instead of IPS.

\section{Proof Complexity Characterization of VP $\neq$ VNP}

We show that IPS cannot efficiently prove that the algebraic circuit class separation VP $\neq$ VNP is hard to prove in IPS. Note that this result is unconditional: IPS unconditionally does not have 
polynomial-size refutations of the statement asserting the existence of short IPS refutations for $\mathrm{VP}=\mathrm{VNP}$. On the other hand, if VP $\neq$ VNP is in fact easy to prove in IPS or if VP $=$ VNP, then the result is less interesting, since then the result stems simply from soundness of IPS. Similarly, if the IPS refutations of VP $=$ VNP are of exponential degree, then the result becomes trivially true again, since there $i s$ a polynomial-size refutation of our formulation of VP $=$ VNP (since the formulation is exponential in itself). The more interesting scenario is under the reasonable assumption that indeed algebraic circuit class separations are hard to establish in polynomial-degree IPS, and in this case we show that this fact would not have efficient proofs in IPS.

\subsection{Formalisations}

\subsubsection{CNF Encoding of Algebraic Circuit Equations}

We are going to work at times with IPS that does not have the boolean axioms for all variables (but only for some variables that will be explicitly specified), namely algebraic IPS, denoted IPS ${ }^{\text {alg }}$. If we show that it is hard to refute that IPS without the boolean axioms has small refutations of some statements, then we also show that it is hard to refute that an IPS with the boolean axioms has small refutations of this statement (otherwise, a short refutation of the existence of IPS refutation with boolean axioms would imply a short refutation of the existence of a refutation in a weaker system). Hence in what follows even when we discuss CNF formulas translated to the algebraic setting we shall write precisely what the boolean axioms that we add to the formulas are.

For an algebraic circuit $C$ and $b$ a field element, we call $C=b$ a circuit equation (we sometimes use the same notion for equations between two circuits). We work over a finite field $\mathbb{F}_{q}$. This is necessary in our argument to be able to switch between formulas in CNF and algebraic circuit equations. Recall that a formula in CNF ("a CNF" for short) is a conjunction of clauses, where a clause is a disjunction of literals, and literals are variables or their negation. The algebraic translation of a formula in CNF is defined according to Definition 2.3. When we work with CNF formulas in IPS we assume that the CNF formulas are translated according to Definition 2.3. The size of objects like circuits, circuit equations, sets of circuit equations and formulas in CNF are denoted by $|\cdot|$ (where "." is replaced by the respective object).

Definition 3.1 (Algebraic extension axioms and unary bits). Given a circuit $C$ and a gate $g$ in $C$ we call the equation

$$
x_{g}=\sum_{i=0}^{q-1} i \cdot x_{g i}
$$

the algebraic extension axiom of $g$, with each variable $x_{g i}$ being the ith unary-bit of $g$.

Note that if the unary-bits of $g$ are taken over $\{0,1\}$ and assuming that $x_{g i}=1$ for precisely one $0 \leq i \leq q-1$, then $x_{g}=i$ iff $x_{g i}=1$. Note also that the unary-bits are disjoint from the (algebraic input) variables of the circuit $C$.

Definition 3.2 (Plain CNF encoding of algebraic circuits; $\operatorname{cnf}(C(\bar{x}))$ ). Let $C(\bar{x})$ be a circuit in the variables $\bar{x}$. The plain $\boldsymbol{C N F}$ encoding of the circuit $C(\bar{x})$ denoted $\operatorname{cnf}(C(\bar{x}))$ consists of the following CNFs in the unary-bits variables of all the gates in $C$ (and only in the unary-bit variables):

1. If $x_{i}$ is an input gate in $C$, the plain $C N F$ encoding of $C$ uses the variables $x_{x_{i} 0}, \ldots, x_{x_{i}(q-1)}$ that are the unary-bits of $x_{i}$, and contains the clauses that express that precisely one unary-bit 
is 1 and all other unary-bits are 0: ${ }^{7}$

$$
\bigvee_{j=0}^{q-1} x_{x_{i} j} \wedge \bigwedge_{j \neq \ell \in\{0, \ldots, 1\}}\left(\neg x_{x_{i} j} \vee \neg x_{x_{i} \ell}\right)
$$

2. If $\alpha \in \mathbb{F}_{q}$ is a scalar input gate in $C$, the plain $C N F$ encoding of $C$ contains the $\{0,1\}$ constants corresponding to the unary-bits of $\alpha$. These constants are used when they are fed to (translations of) gates according to the wiring of $C$ in item 4.

3. For every gate $g$ in $C(\bar{x})$ and every satisfying assignment $\bar{\alpha}$ to the plain $C N F$ encoding, the corresponding unary-bit $x_{g i}$ evaluates to 1 iff the value of $g$ is $i \in\{0, \ldots, q-1\}$ (when the algebraic inputs $\bar{x} \in\left(\mathbb{F}_{q}\right)^{*}$ to $C(\bar{x})$ take on the values corresponding to the boolean assignment $\bar{\alpha}$; “*” here means the Kleene star). This is ensured with the following equations: if $g=u \circ v$ is an internal gate in $C$ (including the output gate, but excluding the input gates of $C$ ), for $\circ \in\{+, \times\}$, we have a CNF $\varphi_{g}$ in the unary-bits variables of $g, u, v$ that is satisfied by an assignment precisely when the output unary-bits of $g$ get their correct values based on the (constant-size) truth table of $\circ$ over $\mathbb{F}_{q}$ and the input unary-bits of $u, v$ (we ensure that if more than one unary-bit is assigned 1 in any of the unary-bits of $g, u, v$ then the CNF is unsatisfiable).

4. For every unary-bit variable $x_{g i}$ we have the boolean axiom (recall we write these boolean axioms explicitly since we are going to work with IPS $^{\text {alg }}$ ):

$$
x_{g i}^{2}-x_{g i}=0 \text {. }
$$

We now define the same encoding, only for a circuit equation instead of merely a circuit, namely the CNF is satisfied by an assignment to the unary-bits precisely when the gates encode correctly the computation of the circuit and additionally the output gate evaluates to zero.

Definition 3.3 (Plain CNF encoding of algebraic circuit equations; $\operatorname{cnf}(C(\bar{x})=0)$ ). Let $C(\bar{x})=0$ be a circuit equation in the variables $\bar{x}$. The plain $\boldsymbol{C N F}$ encoding of the circuit equation $C(\bar{x})=0$ denoted $\operatorname{cnf}(C(\bar{x})=0)$ consists of the plain $C N F$ encoding from Definition 3.2 in the unary-bits variables of all the gates in $C$ (and only in the unary-bit variables), together with the equations

$$
x_{g_{\text {out } 0} 0}=1 \text { and } x_{g_{\text {out }} i}=0, \text { for all } i=1, \ldots, q-1,
$$

which express that $g_{\text {out }}=0$, where $g_{\text {out }}$ is the output gate of $C$.

Definition 3.4 (Extended CNF encoding of a circuit equation (circuit, resp.); ecnf $(C(\bar{x})=0)$ (ecnf $(C(\bar{x}))$, resp.)). Let $C(\bar{x})$ be a circuit in the $\bar{x}$ variables over the finite field $\mathbb{F}_{q}$. Then the extended $\boldsymbol{C N F}$ encoding of the circuit equation $C(\bar{x})=0$ (circuit $C(\bar{x})$, resp.), in symbols ecnf $\left(C(\bar{x})=0\right.$ ) (ecnf $(C(\bar{x}))$, resp.), is defined to be a set of algebraic equations over $\mathbb{F}_{q}$ in the variables $x_{g}$ and $x_{g 0}, \ldots, x_{g q-1}$ which are the unary-bit variables corresponding to node $g$ in $C$, that consists of:

1. the plain $C N F$ encoding of the circuit equation $C(\bar{x})=0$ (circuit $C(\bar{x})$, resp.), namely, $\operatorname{cnf}(C(\bar{x})=0)(\operatorname{cnf}(C(\bar{x}))$, resp.); and

2. the algebraic extension axiom of $g$, for every gate $g$ in $C$.

\footnotetext{
${ }^{7}$ This conditions is needed only for inputs. For internal gates the CNFs expressing the truth table for the gate will make sure that only one output unary-bit takes on the value one.
} 
Notice that the extended CNF encoding is not formally a CNF since it uses the algebraic extension axioms which are not clauses. Also, note that the extended CNF encoding does not contain the boolean axioms for the algebraic extension variables $x_{g}$, for $g$ a gate in $C$, as these variables are meant to range over all $\mathbb{F}_{q}$ and not merely $\{0,1\}$ (while it does contain the boolean axioms for the unary-bit variables).

Since we work with extension variables for each gate in a given circuit equation $C(\bar{x})=0$, it is more convenient to express circuit equations as a set of equations that correspond to the straight line program of $C(\bar{x})$ (which is an equivalent in strength formulation to algebraic circuits):

Definition 3.5 (Straight line program (SLP)). An $\boldsymbol{S L P}$ of a circuit $C(\bar{x})$ is a sequence of equations between variables such that the extension variable for the output gate computes the value of the circuit assuming all equations hold. Formally, we choose any topological order $g_{1}, g_{2}, \ldots, g_{i}, \ldots, g_{|C|}$ on the gates of the circuit $C$ (that is, if $g_{j}$ has a directed path to $g_{k}$ in $C$ then $j<k$ ) and define the following set of equations to be the SLP of $C(\bar{x})$ :

$g_{i}=g_{j} \circ g_{k}$ for $\circ \in\{+, \times\}$ iff $g_{i}$ is a $\circ$ gate in $C$ with two incoming edges from $g_{j}$ and $g_{k}$.

An SLP representation of a circuit equation $C(\bar{x})=0$ means that we add to the SLP above the equation $g_{|C|}=0$, where $g_{|C|}$ is the output gate of the circuit.

Using the concept of extended CNF encoding we can show how to efficiently go within IPS from a circuit equation written as a set of equations for the corresponding SLP to a CNF, and vice versa. The idea is to augment the SLP of $C(\bar{x})=0$ with $x_{g}=\sum_{i=0}^{q-1} i \cdot x_{g i}$ which is the algebraic extension axiom of $g$, for every gate $g$ in $C$. We show that, efficiently in IPS, we can go from this representation of $C(\bar{x})=0$ to its extended CNF encoding, and vice versa.

Proposition 3.6 (Translating between extended CNFs and circuit equations). Let $\mathbb{F}$ be a finite field, and let $C(\bar{x})$ be a circuit in the $\bar{x}$ variables over $\mathbb{F}$ that is written as a set of equations corresponding to the SLP of $C(\bar{x})$. Then, the following both hold (recall that $\frac{*}{\mathrm{IPS}^{\text {alg }}}$ means polynomial-size proofs):

$$
\begin{gathered}
\operatorname{ecnf}(C(\bar{x})=0) \frac{*}{\mathrm{IPS}^{\text {alg }}} C(\bar{x})=0 \\
\left\{x_{g}=\sum_{i=0}^{q-1} i \cdot x_{g i}: g \text { a node in } C\right\}, \\
\left\{x_{g i}^{2}-x_{g i}=0: g \text { is a node in } C, 0 \leq i<q\right\}, \quad \frac{*}{\operatorname{IPS} \text { alg }} \operatorname{ecnf}(C(\bar{x})=0) \\
\left\{\sum_{i=0}^{q-1} x_{g i}=1: g \text { is a node in } C\right\}, C(\bar{x})=0
\end{gathered}
$$

Note that in eq. 3 the first set of axioms from the left are the algebraic extension axioms, the second are the boolean axioms for the unary bits, and the bottom left set of axioms expresses that each gate has only one unary-bit equal to 1 .

The proof uses the fact that over finite fields the truth table of each algebraic gate is easy to describe and can be reasoned about efficiently.

Proof: The proof of eq. 2 is as follows. For every gate $g=u \circ v$ (for $\circ \in\{+, \times\}$ ) we have the corresponding truth table CNF for $\circ$ over $\mathbb{F}_{q}$ that is satisfied only when the unary-bits of gate 
$g$, denoted collectively as $\overline{x_{g i}}$, correspond to the correct output of $g$ given the unary-bits of $u, v$ as inputs, denoted accordingly by $\overline{x_{u i}}, \overline{x_{v i}}$, respectively. Denote this CNF by plus $\left(\overline{x_{g i}}, \overline{x_{u i}}, \overline{x_{v i}}\right)$ or times $\left(\overline{x_{g i}}, \overline{x_{u i}}, \overline{x_{v i}}\right)$, for $\circ=+, \circ=\times$, respectively. In ecnf $(C(\bar{x})=0)$ we also have the algebraic extension axioms for $g, v, u$. It remains to prove that from

$$
\begin{gathered}
\operatorname{plus}\left(\overline{x_{g i}}, \overline{x_{u i}}, \overline{x_{v i}}\right), \text { and } \\
x_{g}=\sum_{i=0}^{q-1} i \cdot x_{g i}, \quad x_{u}=\sum_{i=0}^{q-1} i \cdot x_{u i}, \quad x_{v}=\sum_{i=0}^{q-1} i \cdot x_{v i}
\end{gathered}
$$

we can derive with a constant-size IPS derivation

$$
x_{g}=x_{u}+x_{v},
$$

and similarly for the product gate. This suffices to conclude the proof because the collection of all eq. 6 for all gates $g$ in $C$ are precisely the SLP of $C(\bar{x})$.

We prove the case for plus gates (the case of product gates is similar). Observe that the boolean axioms for the unary-bits and eq. 4 semantically imply (over $\mathbb{F}_{q}$ )

$$
\sum_{i=0}^{q-1} i \cdot x_{g i}=\sum_{i=0}^{q-1} i \cdot x_{u i}+\sum_{i=0}^{q-1} i \cdot x_{v i} .
$$

By implicational completeness of IPS over 0-1 assignments (this is proved by induction on the number of variables, essentially trying out all possible 0-1 assignments to the variables) we get that from the boolean axioms and eq. 4 we have a constant-size derivation of eq. 7 (since the number of variables involved is constant and all variables involved have their corresponding boolean axioms). By substituting eq. 5 in eq. 7 we finish.

The proof of eq. 3 is identical, only that we go in the other direction: we show that from eq. 6 and eq. 5 we can derive efficiently eq. 4 . For that purpose, replace in eq. 6 the right hand sides of eq. 5. This substitution, together with the boolean axioms for the unary-bits that are given as assumptions, semantically imply eq. 4, and by implicational completeness of IPS over 0-1 assignments (since this derivation involves only unary-bit variables, hence all variables have their own boolean axioms) we conclude the case for the plus gate. The case of the product gate is similar.

We wish to speak about CNF formulas and not extended CNF formulas. This is necessary since the work of [GP18], showing that an IPS lower bound implies VNP $\neq$ VP, is known to hold only for lower bounds against CNF formulas.

We have the following simple proposition:

Proposition 3.7. Let $C(\bar{x})=0$ be a circuit equation over $\mathbb{F}_{q}$. Then, $C(\bar{x})=0$ is unsatisfiable over $\mathbb{F}_{q}$ iff $\operatorname{cnf}(C(\bar{x})=0)$ is an unsatisfiable $C N F$ iff ecnf $(C(\bar{x})=0)$ is an unsatisfiable set of equations over $\mathbb{F}_{q}$.

Proof: ecnf $(C(\bar{x})=0)$ only adds to $\operatorname{cnf}(C(\bar{x})=0)$ the algebraic extension axioms in the new variables $x_{g}$, for every gate $g$ in $C$, where $x_{g}$ does not occur anywhere else in ecnf $(C(\bar{x})=0)$ (recall there are no boolean axioms for the variables $x_{g}$ ). Hence, every 0-1 satisfying assignment to $\operatorname{cnf}(C(\bar{x})=0)$ (recall that $\operatorname{cnf}(C(\bar{x})=0)$ includes the boolean axioms for all of its variables hence a satisfying assignment to it must be a $0-1$ assignment) can be extended to a satisfying assignment of ecnf $(C(\bar{x})=0)$, and every satisfying assignment of ecnf $(C(\bar{x})=0)$ is also a satisfying assignment to $\operatorname{cnf}(C(\bar{x})=0)$ (when restricted to the variables in $\operatorname{cnf}(C(\bar{x})=0)$ ).

The fact that $C(\bar{x})=0$ is unsatisfiable over $\mathbb{F}_{q}$ iff $\operatorname{cnf}(C(\bar{x})=0)$ is an unsatisfiable CNF follows from the construction of $\operatorname{cnf}(C(\bar{x})=0)$ (Definition 3.3). 
Corollary 3.8. If ecnf $(C(\bar{x})=0)$ is unsatisfiable over $\mathbb{F}_{q}$ then it has an IPS alg refutation in VNP.

Proof: By Prop. $3.7 \mathrm{cnf}(C(\bar{x})=0)$ is an unsatisfiable CNF. By [GP18] every unsatisfiable CNF has an IPS ${ }^{\text {alg }}$ refutation computable in VNP, namely a refutation that does not use the boolean axioms. Since by definition $\operatorname{cnf}(C(\bar{x})=0) \subseteq \operatorname{ecnf}(C(\bar{x})=0)$, the IPS alg refutation of $\operatorname{cnf}(C(\bar{x})=0)$ is also a refutation of ecnf $(C(\bar{x})=0)$. (Note that in this IPS ${ }^{\text {alg }}$ refutation we can assume we do not use the boolean axioms of the unary-bit variables from item 4 in Definition 3.2, even though we added these axioms explicitly to the system of equations.)

\subsubsection{Encoding Universal Circuits}

To express in the theory that a circuit computes a certain polynomial we will use the concept of a universal circuit as introduced by Raz [Raz10]. A universal circuit is an algebraic circuit that, loosely speaking, embeds all possible circuits of a certain size. More precisely, a universal circuit for the class of polynomials in $\mathbb{F}[\bar{x}]$ that have algebraic circuits of size at most $t$ is a circuit $U(\bar{x}, \bar{w})$ with two sets of variables $\bar{x}$ and $\bar{w}$, such that $f(\bar{x}) \in \mathbb{F}[\bar{x}]$ has circuit of size at most $t$ iff there is a fixed choice of values $\bar{\alpha}$ to $\bar{w}$ for which $U(\bar{x}, \bar{\alpha})=f(\bar{x})$ (as a polynomial identity). Intuitively one can think of the $\bar{w}$ variables as the circuit variables, while the $\bar{x}$ variables are the algebraic variables of the circuit that is encoded by the $\bar{w}$ variables. Formally, $\bar{w}$ describe the edge labels put on edges of a universal circuit.

Raz [Raz10] showed the existence of small algebraic universal circuits for homogeneous polynomials (see also an intuitive description in [SY10]):

Theorem 3.9 (Existence of universal circuits for homogeneous polynomials; Raz [Raz10]). Let $\mathbb{F}$ be a field and $\bar{x}$ be $n$ variables, and let $\mathcal{C}_{t, d}^{\text {hom }}$ denote the class of all homogeneous polynomials of total degree exactly d in $\mathbb{F}[\bar{x}]$ that have algebraic circuits of size at most $t$. Then there is a circuit $U(\bar{x}, \bar{w}) \in \mathbb{F}[\bar{x}, \bar{w}]$ of size $O\left(d t^{4}\right)$ and syntactic-degree $d$ such that $\bar{w}$ are $t$ variables which are disjoint from $\bar{x}$, that is universal for $\mathcal{C}_{t, d}^{\text {hom }}$ in the following sense: $f(\bar{x}) \in \mathcal{C}_{t, d}^{\text {hom }}$ iff there exists $\bar{\alpha} \in \mathbb{F}^{t}$ such that $U(\bar{x}, \bar{\alpha})=f(\bar{x})$.

The idea behind the proof of Thm. 3.9 is to provide a normal form for circuits: every syntactic homogeneous circuit of degree $d$ is reduced with a small increase in size to a normal form in which different choices of edge labels determine the polynomial the circuit computes.

Since we do not necessarily work with homogeneous circuits and polynomials we will assume that the universal circuit $U(\bar{x}, \bar{w})$ is in fact universal for general (non-homogeneous) circuits of a given degree $d$ and size $t$. We can assume this by defining a universal circuit as a sum of the universal circuits for each homogeneous degree as follows (this does not constitute a restriction when considering polynomial degrees, since the classical result of Strassen [Str73] shows that every algebraic circuit computing a degree at most $d$ polynomial can be written as a sum of homogeneous circuits for each of the homogeneous components of the polynomial; where in addition each homogeneous circuit has size polynomially bounded in the original circuit size).

Definition 3.10. The universal circuit for degree $d$ and size $t$ circuits is defined as:

$$
U(\bar{x}, \bar{w})=\sum_{i=0}^{d} U_{i}(\bar{x}, \bar{w}),
$$

where $U_{i}(\bar{x}, \bar{w})$ is the universal circuit for homogeneous $\bar{x}$-polynomials of $i$ degree $\mathcal{C}_{t, i}^{\text {hom }}$ and where the $\bar{w}$-variables in each distinct $U_{i}(\bar{x}, \bar{w})$ are pairwise disjoint (namely, no variable $w_{l}$ appears in both $U_{i}(\bar{x}, \bar{w})$ and $U_{j}(\bar{x}, \bar{w})$ for $\left.i \neq j\right)$. 
By Thm. 3.9 the size of $U(\bar{x}, \bar{w})$ is $\sum_{i=0}^{d} O\left(i t^{4}\right)=O\left(d^{2} t^{4}\right)$.

In our formalisation we are going to express that a circuit computes a polynomial in $\mathbb{F}[\bar{x}]$ by stating that the coefficient vector of a universal circuit described by edge variables $\bar{w}$ equals some fixed vector in $\mathbb{F}^{N}$, with $N$ being the total number of possible $\bar{x}$-monomials in a degree at most $d$ polynomial with a given number of variables, usually $n$. Hence, we represent a circuit of size $s$ using $s$ variables $\bar{w}$ for the $s$ edges in the circuit $U(\bar{x}, \bar{w})$. Note that $U(\bar{x}, \bar{w})$ is a circuit in both $\bar{x}, \bar{w}$, while our formalisation will not use in the end the $\bar{x}$-variables: each $\bar{x}$-monomial will be encoded as a polynomial in the $\bar{w}$-variables representing the coefficient of this $\bar{x}$-monomial. In other words, the coefficient vector of the polynomial in the $\bar{x}$-variables computed by $U(\bar{x}, \bar{w})$ is a vector of $N$ polynomials in the $\bar{w}$ variables, where $N$ is the total number of $\bar{x}$-monomials.

We need to show how to compute given $U(\bar{x}, \bar{w})$ the coefficient of an $\bar{x}$-monomial $M$ as a polynomial in the edge variables $\bar{w}$. Such a polynomial is denoted $\operatorname{Coeff}_{M}(U(\bar{x}, \bar{w}))$.

Let $f(\bar{x}, \bar{w}) \in \mathbb{F}[\bar{x}, \bar{w}]$ be a polynomial, and let $M=\prod_{i \in I} x_{i}^{\alpha_{i}} \cdot \prod_{j \in J} w_{j}^{\beta_{j}}$ be a monomial in $f(\bar{x}, \bar{w})$, for some $\alpha_{i}, \beta_{i} \in \mathbb{N}$ (where $0 \in \mathbb{N}$ ). Then, we call $\sum_{i \in I} \alpha_{i}$ the $\bar{x}$-degree of $M$.

Definition 3.11 $\left(\operatorname{Coeff}_{M}(\cdot)\right)$. Let $f(\bar{x}, \bar{w})$ be a polynomial in $\mathbb{F}[\bar{x}, \bar{w}]$ in the disjoint sets of variables $\bar{x}, \bar{w}$. Let $M$ be an $\bar{x}$-monomial of degree $j$. Then, $\operatorname{Coeff}_{M}(f(\bar{x}, \bar{w}))$ is the (polynomial) coefficient in $\mathbb{F}[\bar{w}]$ (that is, in the $\bar{w}$-variables only) of $M$ in $f(\bar{x}, \bar{w}) .{ }^{8}$

Note that $f(\bar{x}, \bar{w})=\sum_{M_{i}} M_{i} \cdot$ Coeff $_{M_{i}}(f(\bar{x}, \bar{w}))$, where the $M_{i}$ 's are all possible $\bar{x}$-monomials of degree at most $d$, for $d$ the maximal $\bar{x}$-degree of a monomial in $f(\bar{x}, \bar{w})$.

Proposition 3.12 (Computation of coefficients). Let $f(\bar{x}, \bar{w}) \in \mathbb{F}[\bar{x}, \bar{w}]$ be a polynomial in $\mathbb{F}[\bar{x}, \bar{w}]$ in the disjoint sets of variables $\bar{x}, \bar{w}$. Suppose that $M$ is an $\bar{x}$-monomial of degree $d$, and assume that there is an algebraic circuit computing $f(\bar{x}, \bar{w})$ of size $s$ and syntactic-degree $\ell$. Then, there is a circuit of size $O\left(7^{d} \cdot s\right)$ computing $\operatorname{Coeff}_{M}(f(\bar{x}, \bar{w}))$ of syntactic-degree $\ell^{O(1)}$.

Proof: For a variable $x_{i}$ we show how to construct circuits that compute the polynomials $g, h$, respectively, such that $x_{i} \cdot g+h=f$, with $h$ having no occurrences of $x_{i}$ (i.e., $x_{i}$ does not appear with a positive power in any monomial in $h$ ). Thus, $g$ is the polynomial coefficient of $x_{i}$ in $f$. We then continue in this manner to extract the polynomial coefficient of $M$ in $f$. Each such circuitconstruction increases the size of $f$ by a constant factor of 7 according to the claim below. Hence, using $d$ such iterations for each of the $d$ variables in $M$ we shall get a $O\left(7^{d} \cdot s\right)$-size circuit $D$ computing the polynomial coefficient of $M$ in $f(\bar{x}, \bar{w})$. However, this polynomial coefficient may contain also monomials in both the $\bar{x}$ and $\bar{w}$ variables (while by Definition 3.11 it should not), and to eliminate these monomials we simply assign zeroes to all $\bar{x}$-variables in $D$.

It remains to prove the following claim (recall that $\widehat{C}$ is the polynomial computed by a circuit $C$ and that $|C|$ is the size of $C$; we denote by $\widehat{C}(\bar{x}) \uparrow_{x_{i}=0}$ the polynomial $\widehat{C}(\bar{x})$ where $x_{i}$ is assigned $0)$.

Claim 3.13. Let $C(\bar{x})$ be a circuit of syntactic-degree $\ell$ in the $\bar{x}$ variables over the field $\mathbb{F}$. Then, for every variable $x_{i}$ there is a circuit of size $7|C|$ and syntactic-degree $\ell^{O(1)}$ that computes the polynomial $g(\bar{x})$, such that $\widehat{C}(\bar{x})=x_{i} \cdot g(\bar{x})+\widehat{C}(\bar{x}) \uparrow_{x_{i}=0}$.

Proof of claim: The proof is by induction on the circuit size. Denote by $p$ the polynomial computed by $C$ and for every gate $v$ in $C$ denote by $p_{v}$ the polynomial computed at gate $v$.

Denote by $\mathbf{P}_{x_{i}}\left(p_{v}\right)$ the unique polynomial such that $p_{v}=x_{i} \cdot \mathbf{P}_{x_{i}}\left(p_{v}\right)+p_{v} \uparrow_{x_{i}=0}$ (note that $\mathbf{P}_{x_{i}}\left(p_{v}\right)$ can contain $x_{i}$ because $p_{v}$ is not necessarily linear in $\left.x_{i}\right)$. For every gate $v$ in $C$ we add at

\footnotetext{
${ }^{8}$ Note that there can be polynomial coefficients in $\mathbb{F}[\bar{x}, \bar{w}]$ of $M$ that involve also the $\bar{x}$-variables. But we wish to consider the polynomial coefficients in the $\bar{w}$-variables alone. For this reason, in Prop. 3.12 we shall assign zero values to the $\bar{x}$-variables after taking the polynomial coefficient of $M$ in the $\bar{w}$-variables.
} 
most 7 new gates. The gate $v$ itself is duplicated twice so that the first duplicate computes $\mathbf{P}_{x_{i}}\left(p_{v}\right)$ and the second duplicate computes $p_{v} \Upsilon_{x_{i}=0}$.

Base case:

Case 1: $C(\bar{x})=x_{i}$. Then, $\mathbf{P}_{x_{i}}(p):=1$ and $p \uparrow_{x_{i}=0}:=0$.

Case 2: $C(\bar{x})=x_{j}$, for $j \neq i$. Then, $\mathbf{P}_{x_{i}}(p):=0$ and $p \uparrow_{x_{i}=0}:=x_{j}$.

Case 3: $C(\bar{x})=\alpha$, for $\alpha \in \mathbb{F}$. Then, $\mathbf{P}_{x_{i}}(p):=0$ and $p \uparrow_{x_{i}=0}:=\alpha$.

Induction step:

Case 1: $C(\bar{x})=w+u$. Then, $\mathbf{P}_{x_{i}}(p):=\mathbf{P}_{x_{i}}\left(p_{w}\right)+\mathbf{P}_{x_{i}}\left(p_{u}\right)$ and $p \uparrow_{x_{i}=0}:=p_{w} \uparrow_{x_{i}=0}+p_{u} \Upsilon_{x_{i}=0}$.

Case 2: $C(\bar{x})=w \cdot u$. Then,

$$
\begin{gathered}
\mathbf{P}_{x_{i}}(p):=\mathbf{P}_{x_{i}}\left(p_{w}\right) \cdot x_{i} \cdot \mathbf{P}_{x_{i}}\left(p_{u}\right)+\mathbf{P}_{x_{i}}\left(p_{w}\right) \cdot\left(p_{u}\left\lceil x_{i}=0\right)+\mathbf{P}_{x_{i}}\left(p_{u}\right) \cdot\left(p_{w}\left\lceil x_{i}=0\right),\right. \text { and }\right. \\
p \uparrow_{x_{i}=0}:=\left(p_{u} \Upsilon_{x_{i}=0}\right) \cdot\left(p_{w} \Upsilon_{x_{i}=0}\right) .
\end{gathered}
$$

Note that the construction results in a circuit and not a formula, that is, in the induction step above we re-use gates that where already used if necessary (in correspondence with the wiring and re-usage of gates in the original circuit $C$ ). This brings us to a circuit of size at most $7|C|$, and by construction syntactic-degree $\ell^{O(1)}$. $\mathbf{a}_{\text {Claim }}$

By immediate inspection of Claim 3.13 we have:

Corollary 3.14. Let $C(\bar{w})$ be a circuit that contains only the $\bar{w}$-variables and $M$ be an $\bar{x}$-monomial (with $\bar{w}$ and $\bar{x}$ pair-wise disjoint). Then, $C(\bar{w})$ stays intact under the construction of Coeff ${ }_{M}(\cdot)$, namely $\operatorname{Coeff}_{M}(C(\bar{w}))=C(\bar{w})$.

Remark 3.15. It will be important for us that there are small universal circuits, namely that the size of $U(\bar{x}, \bar{w})$ is small, since our hypothesized hard candidates will use a circuit computing $U(\bar{x}, \bar{w})$. The diagonalisation argument works regardless of the size of the instance, only that if the size of the formulas proved is too big, and specifically exponential in the number of variables, we land in the uninteresting case in which there will be no short refutations of the statement $\Phi$ expressing short IPS refutations of VNP $=\mathrm{VP}$, simply because $\Phi$ is satisfiable, namely there are polynomial-size (in the unsatisfiable input $C N F$ ) refutations of VP $=$ VNP. We explain this further in Subsection 3.2.

\subsubsection{Formalising VNP $\neq$ VP}

The class VP is expressed using a universal circuit while the class VNP is expressed explicitly as the coefficient vector of the permanent polynomial, where permanent is known to be complete for VNP [Val79a].

Let $\operatorname{perm}(\bar{x})$ be the permanent polynomial on the variables $\bar{x}$. We are going to encode the negation of VP $\neq$ VNP (since we work with the refutation system IPS, we prove statements by refuting their negations):

Definition 3.16 (Formalisation of $\mathrm{VP}=\mathrm{VNP})$. The formalisation of $\mathrm{VNP}=\mathrm{VP}(t, n, d)$ denoted "VNP $=\mathrm{VP}(t, n, d)$ ", expressing that there is a universal circuit for degree $d \geq n$ and size $t$ circuits that computes the permanent polynomial of dimension $n$ (with $\bar{x}$ being the $n^{2}$ variables of the permanent), is the following set of polynomial equations (in the $\bar{w}$-variables only ${ }^{9}$ ):

$$
\left\{\operatorname{Coeff}_{M_{i}}(U(\bar{x}, \bar{w}))=b_{i}: 1 \leq i \leq N\right\},
$$

\footnotetext{
${ }^{9}$ Recall that $\operatorname{Coeff}_{M}(U(\bar{x}, \bar{w}))$ is a polynomial in the $\bar{w}$-variables only, for $M$ an $\bar{x}$-monomial.
} 
where $\bar{b}=\operatorname{coeffs}(\operatorname{perm}(\bar{x})) \in \mathbb{F}^{N}$ is the coefficient vector of the permanent polynomial of dimension $n, \bar{w}$ are the $t$ edge variables, $\left\{M_{i}\right\}_{i=1}^{N}$ is the set of all possible $\bar{x}$-monomials of degree at most $d$, and $N=\sum_{j=0}^{d}\left(\begin{array}{c}n^{2}+j-1 \\ j\end{array}\right)=2^{O\left(n^{2}+d\right)}$ is the number of monomials of total degree at most $d$ over $n^{2}$ variables.

By Prop. 3.12 the size of each circuit equation in eq. 9 is $O\left(7^{j} \cdot|U(\bar{x}, \bar{w})|\right)=O\left(7^{n} \cdot d^{2} t^{4}\right)$ (with $j \leq d$ the degree of the $\bar{x}$-monomial $M_{i}$ ), meaning that

$$
\text { the size of "VNP }=\mathrm{VP}(t, n, d) \text { " is } O\left(7^{n} \cdot d^{2} t^{4} \cdot N\right)=t^{4} \cdot 2^{O\left(n^{2}+d\right)},
$$

and the syntactic-degree of each circuit equation in eq. 9 is $d^{O(1)}$.

\section{Remark 3.17.}

1. Note that "VNP $=\operatorname{VP}(t, n, d)$ " (which we denote by "VNP $=\mathrm{VP} "$ when we do not care for the parameters $t, n, d)$ does not contain the $\bar{x}$ variables, rather only the $\bar{w}$ variables.

2. The degree $d$ parameter is displayed in "VNP $=\mathrm{VP}(t, n, d)$ " due to technical reasons: we would want the universal circuit that purportedly computes VNP to have unbounded syntactic-degree $d$ (and not merely syntactic-degree $n$ ), because in our diagonalization argument later we will turn this universal circuit and the statement "VNP $=\operatorname{VP}(t, n, d)$ " into the statement asserting that there is a small IPS refutation of degree $d$ (computed by the universal circuit) and not merely an IPS refutation of smaller degree $n$. (It is worth mentioning again that any circuit computing a polynomial of degree $n$ can be converted with only a polynomial increase in size to a circuit of syntactic-degree $n$, following standard homogenization [Str73].)

3. Note that assuming $\mathrm{VNP} \neq \mathrm{VP}$, "VNP $=\mathrm{VP}(t, n, d)$ " is indeed an unsatisfiable set of polynomial equations over $\mathbb{F}_{q}$ for $n$ big enough and $t$ polynomially bounded in $n$ : any assignment for the $\bar{w}$ variables that satisfies "VNP $=\operatorname{VP}(t, n, d)$ " means that there is a $t$-size circuit (induced by the edge variables $\bar{w}$-assignment) that computes the permanent polynomial (in the $\bar{x}$ variables).

\subsubsection{Formalising IPS Refutations}

To express an IPS lower bound as a set of polynomial equations we will formalise the negation of this statement, namely the existence of a small IPS refutation for a specific CNF formula.

Definition 3.18 (IPS refutation predicate $\operatorname{IPS}_{\text {ref }}(t, d, \overline{\mathcal{F}})$ ). Let $\overline{\mathcal{F}}$ be a $C N F$ formula with $m$ clauses and (for simplicity, since we deal with matrix inputs) $n^{2}$ variables $\bar{x}$ written as a set of polynomial equations according to Definition 2.3. Let $U(\bar{x}, \bar{y}, \bar{w})$ be a universal circuit for degree $d$ and size $t$ circuits in the $\bar{x}$ variables and the $m$ placeholder variables $\bar{y}$ (both of these sets of variables are the algebraic variables in a polynomial computed by the universal circuit when assigned field values to the edge labels $\bar{w})$, and the $t$ edge label variables $\bar{w}$. We formalise the existence of a size $t$ and degree $d$ circuit that computes the IPS refutation of $\overline{\mathcal{F}}$, denoted $\operatorname{IPS}_{\text {ref }}(t, d, \overline{\mathcal{F}})$, with the following set of circuit equations (in the $\bar{w}$-variables only):

$$
\begin{aligned}
& \operatorname{Coeff}_{M_{i}}(U(\bar{x}, \mathbf{0}, \bar{w}))=0, \\
\text { Coeff }_{M_{i}}(U(\bar{x}, \overline{\mathcal{F}}, \bar{w}))= & \left\{\begin{array}{ll}
1, & M_{i}=1 \\
0, & \text { otherwise, }
\end{array} \text { (i.e., the constant } 1\right. \text { monomial); }
\end{aligned}
$$

where $i$ ranges over $i \in[N]$ so that $\left\{M_{i}\right\}_{i=1}^{N}$ are the set of all possible $\bar{x}$-monomials of degree at most $d$, and $N=\sum_{j=0}^{d}\left(\begin{array}{c}n^{2}+j-1 \\ j\end{array}\right)=2^{O\left(n^{2}+d\right)}$ is the number of monomials of total degree at most $d$ over $n^{2}$ variables, $\mathbf{0}$ is the all-zero vector of length $m$ (replacing the $\bar{y}$-variables in $U(\bar{x}, \bar{y}, \bar{w})$ ). 
By Prop. 3.12 the size of each circuit equation in eq. 11 and eq. 12 is $O\left(7^{j} \cdot|U(\bar{x}, \bar{y}, \bar{w})| \cdot|\overline{\mathcal{F}}|\right)=$ $O\left(7^{d} \cdot d^{2} t^{4} \cdot|\overline{\mathcal{F}}|\right)$ (with $j \leq d$ the degree of the $\bar{x}$-monomial $M_{i}$ ), meaning that

$$
\text { the size of } \operatorname{IPS}_{\text {ref }}(t, d, \overline{\mathcal{F}}) \text { is } O\left(7^{d} \cdot d^{2} t^{4} \cdot|\overline{\mathcal{F}}| \cdot N\right)=t^{4} \cdot|\overline{\mathcal{F}}| \cdot 2^{O\left(n^{2}+d\right)},
$$

and the syntactic-degree of each circuit equation in eq. 11 and eq. 12 is $d^{O(1)}$.

Encoding an IPS lower bound is simply providing an ostensible unsatisfiable set of polynomials that express the existence of size at most $t$ and degree at most $d$ IPS refutation of a CNF $\overline{\mathcal{F}}$. Thus refuting this set of polynomials amounts to proving an IPS lower bound.

Remark 3.19. Note that our results are interesting only for IPS refutations of degrees $d$ that do not exceed our purported lower bound $t$. More precisely, if $d=\Omega(t)$ then the size of $\lambda=\operatorname{IPS}_{\text {ref }}(t, d, \overline{\mathcal{F}})$ is $2^{\Omega(t)}$ by eq. 13, and since $t$ is the number of variables in $\lambda$ there is a polynomial-size (in $|\lambda|$ ) IPS refutation of $\lambda$. Namely, our candidate $\lambda$ is in fact easy (hence, although the diagonalisation argument still holds if $d=\Omega(t)$, it holds merely because even when $\lambda$ is indeed unsatisfiable it has a polynomial-size IPS refutation since the number of variables is polynomial in its size). See also Sect. 3.2.2 for a discussion of the different parameters.

\subsection{Characterizing VNP $\neq$ VP as a Proof Complexity Lower Bound}

We are now ready to prove our main theorem that IPS cannot prove certain IPS lower bounds assuming VNP $\neq$ VP. The gist of the argument is showing how from a short IPS proof of an IPS lower bound on an unsatisfiable CNF formula one gets a short IPS proof that VP $\neq$ VNP - this can be considered a formalisation in IPS of the Grochow and Pitassi argument [GP18]. Since we work with a refutation system, we will show equivalently that if IPS efficiently refutes the existence of small IPS refutations of some CNF formula, then there is an efficient IPS refutation of VP $=$ VNP. We shall start from the CNF "VP $=$ VNP" and reach a contradiction (in IPS). The idea now is this: if we have the CNF "VNP $=\operatorname{VP}(t, n, d)$ ", meaning that perm $(\bar{x})$ of dimension $n$ is computable by size $t$ circuits (of syntactic-degree $d$ ), then we know in particular, by the completeness of the permanent for VNP and the fact that every unsatisfiable CNF has an IPS refutation computable in VNP (Thm. 2.4), that there is a "projection", namely, an assignment $\bar{a}$ of variables and field elements to $\bar{x}$, such that $\operatorname{perm}(\bar{x} \uparrow \bar{a})$ is the IPS refutation of the CNF "VP $=$ VNP" of some lower dimension. Since the class of size $t$ circuits is closed under assignments we conclude that $\operatorname{perm}(\bar{x}\lceil\bar{a})$ has a size $t$ circuit, that is, there is a small IPS refutation of "VP $=$ VNP" of some lower dimension. But we assumed that IPS can (efficiently) refute the existence of such small IPS refutations for "VP = VNP", and by soundness of IPS we arrived at a contradiction.

We are going to use the following lemma which will allow us to express a proof complexity lower bound on a seemingly stronger ("less satisfiable") statement (and hence, stating such a refutation lower bound seemingly weakens our lower bound statement). In particular we will extend the statement of VP $=$ VNP with additional extension axioms for certain new circuits $C_{i}(\bar{x})$. These extension axioms include the equations for gates in $C_{i}(\bar{x})$ written as SLPs and the algebraic extension axioms for each gate $g$ in $C_{i}(\bar{x})$. On the other hand, these extension axioms do not express that any of the $C_{i}(\bar{x})$ equals any specific value. In particular this means that these additional extension axioms do not add any information to the statement (that is, they do not actually make the statement "less satisfiable"). In return we will be able to state that such additional extension axioms do not make a refutation lower bound against the statement a too strong assumption by itself, in the sense that if we can efficiently refute the stronger (extended) statement then we can efficiently refute also the weaker (non extended) statement: 
Lemma 3.20 (Disjoint extension axioms do not make refutations easier, provably in IPS). Let $\bar{F}$ be a set of circuit equations written as $S L P s, \bar{E}$ be a set of different circuits (not circuit equations ${ }^{10}$ ) each written as an SLP with extension variables that do not appear in $\bar{F}$ for every gate $g$ in the circuits in $\bar{E}$, together with the algebraic extension axioms for each such gate $g$. Then ${ }^{11}$,

$$
\operatorname{cnf}\left(\operatorname{IPS}_{r e f}^{\text {alg }}(t, \operatorname{ecnf}(\bar{F} \cup \bar{E}))\right) \frac{\left.\right|^{*}}{\operatorname{IPS}} \operatorname{cnf}\left(\operatorname{IPS}_{r e f}^{\text {alg }}(t, \operatorname{ecnf}(\bar{F}))\right) .
$$

Proof: The argument is a straightforward substitution to the extension axioms in $\bar{E}$ by some constants that satisfy the extension axioms (the variables in $\bar{E}$ do not occur in $\bar{F}$, and $\bar{E}$ correctly express circuits, and not circuit equations, and so is satisfiable by definition). Note that we must insist on using IPS ${ }^{\text {alg }}$ refutations here in the internal IPS ${ }^{\text {alg }}$ proof predicate (in contrast to IPS), as otherwise the use of boolean axioms for the algebraic extension variables for nodes $g$ in the circuits $\bar{E}$ may make the equations in $\bar{E}$ unsatisfiable (hence, may make the refutation of ecnf $(\bar{F} \cup \bar{E})$ easier than that of ecnf $(\bar{F})$. Similarly, if $\bar{E}$ are circuit equations instead of merely circuits, this may make $\bar{E}$ unsatisfiable, and hence the lemma not true). We omit the details of this formalisation.

Lemma 3.21 (Algebraic extension axioms do not make refutations easier, provably in IPS). For every set of circuit equations $\bar{F}$ written as SLPs the following holds:

$$
\operatorname{cnf}\left(\operatorname{IPS}_{r e f}^{\mathrm{alg}}(t, \operatorname{ecnf}(\bar{F}))\right) \frac{*}{\operatorname{IPS}} \operatorname{cnf}\left(\operatorname{IPS}_{r e f}^{\mathrm{alg}}(t, \operatorname{cnf}(\bar{F}))\right)
$$

Proof: As before, the argument is a substitution. Specifically, consider an IPS refutation of ecnf $(\bar{F})$. If we substitute a variable by a polynomial in a refutation it is still a refutation (namely, a polynomial computing 1) only that we need to make sure that the axioms in the new refutation belong to the set we want to refute, in our case $\operatorname{cnf}(\bar{F})$.

Let $x_{g}$ be a variable in an algebraic extension axiom (Definition 3.1) $x_{g}=\sum_{i=0}^{q-1} i \cdot x_{g i}$ in ecnf $(\bar{F})$ (which formally appears as $x_{g}-\sum_{i=0}^{q-1} i \cdot x_{g i}$ in the IPS refutation). We substitute the variable $x_{g}$ in the refutation by $\sum_{i=0}^{q-1} i \cdot x_{g i}$. Hence, all the algebraic extension axioms vanish (i.e., equal 0 ), and since the variables $x_{g}$ do not occur in any other axiom of ecnf $(\bar{F})$ (though may occur somewhere else in the refutation nevertheless) we get a refutation of the original axioms of $\mathrm{cnf}(\bar{F})$.

As mentioned in Remark 3.19 we are interested in the case where the syntactic degree $d$ does not exceed the size lower bound parameter $t$. Therefore, in what follows

we let $d: \mathbb{N} \rightarrow \mathbb{N}$ be a (monotone) size function $d(t)=t^{\epsilon}$ for some constant $0<\epsilon<1$.

The function $d$ stands for the syntactic-degree of the universal circuit used in either the IPS refutations or the universal circuits for computing the permanent.

Let $\varphi_{r, m, d}^{\mathrm{cnf}}$ denote the CNF encoding of the circuit equation "VNP $=\mathrm{VP}(r, m, d(r))$ " over the field $\mathbb{F}_{q}$ expressing that there are size $r$ and syntactic-degree $d(r)$ circuits for the permanent of dimension $m$ over $\mathbb{F}_{q}$. Let $\Phi_{t, r, m, d}$ denote the $\operatorname{CNF}$ formula $\operatorname{cnf}\left(\operatorname{IPS}_{r e f}\left(t, d, \varphi_{r, m, d}^{\mathrm{cnf}}\right)\right)$ expressing that IPS refutes $\varphi_{r, m, d}^{\mathrm{cnf}}$ in size $t$ and degree $d(t)$ over $\mathbb{F}_{q}$.

\footnotetext{
${ }^{10}$ Recall that this means that we simply add the SLP for the circuit without stating that the circuit's output gate evaluates to any specific value.

${ }^{11}$ Note that ecnf $(\bar{F} \cup \bar{E})=\operatorname{ecnf}(\bar{F}) \cup \operatorname{ecnf}(\bar{E})$ by Definition 3.4, where $\bar{F}$ are circuit equations, and $\bar{E}$ are circuits. Also notice that the variables in ecnf $(\bar{F})$ and in ecnf $(\bar{E})$ are disjoint.
} 
Theorem 3.22 (main). VP $\neq$ VNP over $\mathbb{F}_{q}$ iff the $C N F$ family $\left\{\Phi_{t, r, m, d}\right\}$ does not have polynomialsize IPS refutations infinitely often in the following sense: for every sufficiently large constant $c_{0}$ there exists a constant $c_{1}$ such that for infinitely many ${ }^{12} t, r, m \in \mathbb{N}$ and every sufficiently large constant $c_{2}$, if $t>\left|\varphi_{r, m, d}^{\mathrm{cnf}}\right|^{c_{1}}$ and $m^{c_{1}}<r<m^{c_{2}}, \Phi_{t, r, m, d}$ has no IPS refutation of size at most $\left|\Phi_{t, r, m, d}\right|^{c_{0}}$.

Note that for every proof complexity lower bound $n^{c_{0}}$ that we want to establish, there exists a hard instance stating a proof complexity lower bound of $n^{c_{1}}$ (where $n$ is the size of the formulas refuted or proved).

Proof of direction $(\Leftarrow)$ of Thm. 3.22. This is the easier direction. Assume that there exist sufficiently big constants $c_{0}, c_{1}, c_{2}$ such that for infinitely many $m \in \mathbb{N}$ the family of CNF formulas $\left\{\operatorname{cnf}\left(\operatorname{IPS}_{r e f}\left(s, d, \varphi_{t, m, d}\right)\right)\right\}_{n=1}^{\infty}$ does not have polynomial-size IPS refutations where $t<m^{c_{0}}$ (that is, the purported circuit for the permanent of dimension $m$ is polynomially bounded in $m$ ) and $s<\left|\varphi_{t, m, d}\right|^{c_{1}}$ (that is, the purported size of an IPS refutation of the formula $\varphi_{t, m, d}$ is polynomially bounded in the size of the formula).

If $\left\{\operatorname{cnf}\left(\operatorname{IPS}_{r e f}\left(s, \varphi_{t, m, d}\right)\right)\right\}_{n=1}^{\infty}$ is an unsatisfiable $\operatorname{CNF}$ family for infinitely many $m \in \mathbb{N}$, then by Thm. 2.4 [GP18] VNP $\neq$ VP over $\mathbb{F}_{q}$ (note that the result of [GP18] holds also for lower bounds on IPS without the boolean axioms).

Otherwise, $\left\{\operatorname{cnf}\left(\operatorname{IPS}_{r e f}\left(s, d, \varphi_{t, m, d}\right)\right)\right\}_{n=1}^{\infty}$ is a satisfiable CNF family for infinitely many $m \in \mathbb{N}$. Thus, by Prop. 3.7 the set of polynomial equations $\operatorname{IPS}_{r e f}\left(s, d, \varphi_{t, m, d}\right)$ is satisfiable over $\mathbb{F}_{q}$ for infinitely many $m \in \mathbb{N}$ and hence there is an IPS refutation of $\varphi_{t, m, d}$ for infinitely many $m \in \mathbb{N}$ and by soundness of IPS we get that infinitely often there is no size at most $m^{c_{0}}$ for the permanent of dimension $m$, and we are done.

\subsubsection{Proof of direction $(\Rightarrow)$ of Thm. 3.22}

We work over $\mathbb{F}_{q}$. First, consider the plain CNF encoding $\varphi_{r, m, d}^{\mathrm{cnf}}=\operatorname{cnf}($ "VNP $=\operatorname{VP}(r, m, d(r))$ ") and the extended CNF encoding $\varphi_{r, m, d}^{\text {ecnf }}=\operatorname{ecnf}\left(" \mathrm{VNP}=\operatorname{VP}(r, m, d(r))\right.$ "). Denote by $\varphi_{r, m, d}^{\star}$ the extended $\operatorname{CNF} \varphi_{r, m, d}^{\text {ecnf }}$ together with additional extension axioms $\bar{E}$ for some new set of circuits written as SLPs and a new set of algebraic extension axioms for all the gates in these new circuits that we will specify later (all the variables in these new equations will be disjoint from the original variables in $\left.\varphi_{r, m, d}^{\text {enf }}\right)$. Note that $\bar{E}$ are circuits but not circuit equations, so we have not added new constraints to the original instance (that may make it easier to refute, as we show below). ${ }^{13}$

We assume that:

Assumption 1: VP $\neq$ VNP over $\mathbb{F}_{q}$. Namely, there is no constant $c$ such that for all but constant many $n \in \mathbb{N}$, the permanent polynomial perm $(\bar{x})$ of dimension $n$ has an algebraic circuit of size at most $n^{c}$, over $\mathbb{F}_{q}$.

And we will prove that:

Conclusion 1: Let $c_{0}$ be some sufficiently large constant. There exists a constant $c_{1}$ such that for every sufficiently big constant $c_{2}>c_{1}$, for infinitely many $t, r, m \in \mathbb{N}$ with $t>\left|\varphi_{r, m, d}^{\star}\right|^{c_{1}}$ and $m^{c_{1}}<r<m^{c_{2}}$ the following $\mathrm{CNF}$

$$
\operatorname{cnf}\left(\operatorname{IPS}_{r e f}^{\mathrm{alg}}\left(t, d, \varphi_{r, m, d}^{\star}\right)\right)
$$

\footnotetext{
${ }^{12}$ Here we mean infinitely many distinct $t$ 's, infinitely many distinct $r$ 's and infinitely many distinct $m$ 's.

${ }^{13}$ Note that we cannot assume a priori that the extension axioms $\bar{E}$ are derivable for free in IPS, and this is the reason we need to add them in $\varphi_{r, m, d}^{\star}$. It is reasonable to assume that extension axioms that use new variables do not allow for shorter IPS refutations in a similar way that Extended Frege uses extension axioms and is polynomially equivalent to substitution Frege [Kra95] (however, we are not aware of proofs for this statement).
} 
has no IPS refutations of size at most $\left|\operatorname{cnf}\left(\operatorname{IPS}_{r e f}^{\text {alg }}\left(t, d, \varphi_{r, m, d}^{\star}\right)\right)\right|^{c_{0}}$.

Note that since $\varphi_{r, m, d}^{\star}$ has more constraints than $\varphi_{r, m, d}$, apriori it may happen that IPS cannot efficiently prove that $\varphi_{r, m, d}^{\star}$ is hard for IPS, while IPS can efficiently prove that $\varphi_{r, m, d}$ is hard for IPS. We show that this is not the case, namely, we argue that as long as the set of new axioms $\bar{E}$ in $\varphi_{r, m, d}^{\star}$ have total algebraic circuit-size polynomial in $\left|\varphi_{r, m, d}^{\mathrm{cnf}}\right|$, Conclusion 1 above suffices to conclude the theorem:

Claim 3.23. If Conclusion 1 above holds then also the same conclusion holds when the CNF

$$
\operatorname{cnf}\left(\operatorname{IPS}_{r e f}\left(t, d, \varphi_{r, m, d}^{\mathrm{cnf}}\right)\right)
$$

replaces eq. 16 in Conclusion 1.

Proof of claim: This is established by showing that from a short refutation of eq. 17 we get a short refutation of eq. 16, as follows:

$$
\begin{aligned}
& \operatorname{cnf}\left(\operatorname{IPS}_{r e f}^{\mathrm{alg}}(t, d, \overbrace{\left.\varphi_{r, m, d}^{\mathrm{ecnf}} \cup \bar{E}\right)}^{\varphi_{r, m, d}^{\star}}) \mid \frac{s}{{ }_{\mathrm{IPS}}(1)} \operatorname{cnf}\left(\operatorname{IPS}_{r e f}^{\mathrm{alg}}\left(t, d, \varphi_{r, m, d}^{\mathrm{ecnf}}\right)\right) \quad \text { by Lemma } 3.20\right. \\
& \frac{{ }_{s} O(1)}{\operatorname{IPS}} \operatorname{cnf}\left(\operatorname{IPS}_{r e f}^{\mathrm{alg}}\left(t, d, \varphi_{r, m, d}^{\mathrm{cnf}}\right)\right) \quad \text { by Lemma } 3.21 \\
& \frac{{ }_{s}^{O(1)}}{\operatorname{IPS}} \operatorname{cnf}\left(\operatorname{IPS}_{r e f}\left(t, d, \varphi_{r, m, d}^{\mathrm{cnf}}\right)\right) \text { because IPS is a subsystem of IPS }{ }^{\text {alg }} \text {, }
\end{aligned}
$$

where the last equality is because IPS is a subsystem of IPS ${ }^{\text {alg }}$, and where $s$ is the size of $\operatorname{cnf}\left(\operatorname{IPS}_{r e f}^{\text {alg }}\left(t, d, \varphi_{r, m, d}^{\text {ecnf }} \cup \bar{E}\right)\right)$ and the constant power in the polynomial $s^{O(1)}$ is assumed without loss of generality to be small enough (or else we choose the other constant and specifically $c_{3}$ as below to be big enough).

Notice that to invoke Lemma 3.20 and Lemma 3.21 we need to work with IPS ${ }^{\text {alg }}$ and not IPS. - Claim

We are going to describe the general scheme of the argument first, and then prove separately Lemma 3.25 which is a sort of formalisation of Grochow-Pitassi result within IPS, in what follows.

Scheme of the main argument. Under Assumption 1 above, let $c_{3}$ be some fixed constant (that is derived from the polynomial-size proof in Prop. 3.26), let $c_{0}$ be any constant. Suppose that $t, r, m \in \mathbb{N}$ are such that (recall that " $\frac{s, d}{\text { IPS }}$ " means size $s$ and degree $d$ IPS proofs):

$$
\underbrace{\operatorname{cnf}\left(\operatorname{IPS}_{r e f}^{\text {alg }}(t, d, \overbrace{\varphi_{r, m, d}^{\text {ecnf }}}^{\sigma})\right.}_{\lambda}||^{|\lambda|^{c_{0}, d(|\lambda|)}} 1=0,
$$

where $\varphi_{r, m, d}^{\text {ecnf }}$ is unsatisfiable over $\mathbb{F}_{q}$ by Assumption 1 (for sufficiently big $r, m$ and when $r<m^{c_{2}}$ for a sufficiently big constant $c_{2}$ that we pick in what follows), and moreover eq. 18 is by an assumption that there are i.o. short, degree $d(|\lambda|)$ refutations for the statement $\lambda$ that expresses there are short refutations of $\varphi_{r, m, d}^{\mathrm{ecnf}}$.

Note that if this latter assumption about $t, r, m$ and eq. 18 is incorrect then we finish our proof, since then there exists a constant $c_{1}$ such that for a sufficiently big constant $c_{2}>c_{1}$, and where 
$t>\left|\varphi_{r, m, d}^{\text {ecnf }}\right|^{c_{1}}$ and $m^{c_{1}}<r<m^{c_{2}}, \operatorname{cnf}\left(\operatorname{IPS}_{r e f}^{\text {alg }}\left(t, d, \varphi_{r, m, d}^{\text {ecnf }}\right)\right)$ has no degree $d(|\lambda|)$ IPS refutations of size at most $\left|\operatorname{cnf}\left(\operatorname{IPS}_{r e f}\left(t, d, \varphi_{r, m, d}^{\mathrm{enn}}\right)\right)\right|^{c_{0}}$ (and assuming $c_{0}$ is big enough, there is no IPS refutation of size at most $\left|\operatorname{cnf}\left(\operatorname{IPS}_{r e f}\left(t, d, \varphi_{r, m, d}^{\star}\right)\right)\right|^{c_{0}}$ of $\operatorname{cnf}\left(\operatorname{IPS}_{r e f}\left(t, d, \varphi_{r, m, d}^{\star}\right)\right)$ as well).

Let

$$
\gamma=\varphi_{t, k, d}^{\star} \quad \text { and } \quad k=6\left(\ell+N^{\prime}\right),
$$

where $\ell, N^{\prime}$ are the number of variables and clauses in $\varphi_{r, m, d}$, respectively, and assume that eq. 18 holds and

$$
t>k^{c_{1}}
$$

for a constant $c_{1}$ that we pick big enough so that

$$
|\gamma|^{c_{3}}+|\lambda|^{c_{0}}=|\gamma|^{c_{3}}+|\gamma|^{c^{\prime} \cdot c_{0}}<|\gamma|^{c_{1}}
$$

(we use here that $|\lambda|=\left.|\gamma|\right|^{\prime}$ for some constant $c^{\prime}$, by eq. 10 and eq. 13); and moreover, as before, $\varphi_{t, k, d}^{\star}$ is $\varphi_{t, k, d}^{\text {ecnf }}$ augmented with additional extension axioms $\bar{E}$ for some new set of circuits written as SLPs and a new set of algebraic extension axioms for all the gates in these new circuits that we will specify later (all the variables in these new equations will be disjoint from the original variables in $\left.\varphi_{t, k, d}^{\text {ecnf }}\right)$.

In Lemma 3.25 in the sequel we construct an $\operatorname{IPS}^{\text {alg }}$ derivation of $\operatorname{IPS}_{r e f}^{\text {alg }}\left(t, d, \varphi_{r, m, d}^{\mathrm{ecnf}}\right)$ from $\varphi_{t, k, d}^{\star}$ of size at most $|\gamma|^{c_{3}}$ and degree at most $d(|\lambda|)$, assuming that $\varphi_{r, m, d}^{\mathrm{ecnf}}$ is indeed unsatisfiable (which, as mentioned above, follows from our Assumption 1 and the fact that $r, m$ are big enough and $r<m^{c_{2}}$ for a sufficiently big constant $c_{2}>c_{1}$ ), from which we can conclude, using eq. 18, that:

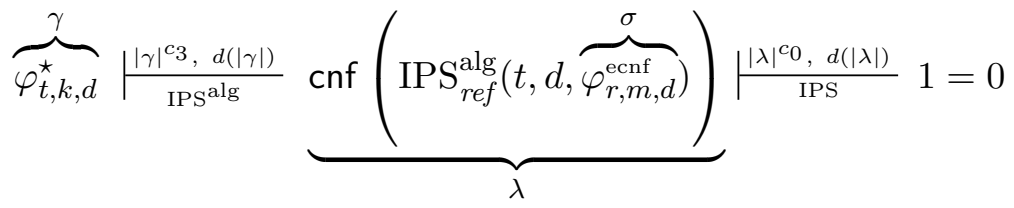

(note that for a CNF $T$, if we have $T \frac{s}{\operatorname{IPS}} 1=0$ we have also $\left.T\right|_{\text {IPSalg }} ^{s} 1=0$, because all variables in the CNF $T$ have boolean axioms explicitly added to $T$ by Definition 3.3, hence we have $\left.\varphi_{t, k, d}^{\star} \frac{\left.|| \gamma\right|^{c 3}, d(|\gamma|)}{\operatorname{IPS} \text { alg }} \operatorname{cnf}\left(\operatorname{IPS}_{r e f}^{\text {alg }}\left(t, d, \varphi_{r, m, d}^{\text {ecnf }}\right)\right) \frac{|\lambda|^{c_{0}, d(|\lambda|)}}{\operatorname{IPS}^{\text {alg }}} 1=0\right)$.

By eq. 22, eq. 20 means that there exists a $w$ sized and degree $d(|\gamma|)$ IPS ${ }^{\text {alg }}$ refutation of $\varphi_{t, k, d}^{\star}$ whenever $w \geq|\gamma|^{c_{1}}$, namely $\operatorname{IPS}_{r e f}^{\text {alg }}\left(w, d, \varphi_{t, k, d}^{\star}\right)$ is satisfiable over $\mathbb{F}_{q}$. Thus, by Prop. 3.7 $\operatorname{cnf}\left(\operatorname{IPS}_{r e f}^{\text {alg }}\left(w, d, \varphi_{t, k, d}^{\star}\right)\right)$ is a satisfiable CNF formula, and so by soundness of IPS:

$$
\operatorname{cnf}\left(\operatorname{IPS}_{r e f}^{\mathrm{alg}}(w, d, \overbrace{\varphi_{t, k, d}^{\star}}^{\gamma})\right) \downarrow_{\operatorname{IPS}} 1=0 .
$$

In other words, we have concluded that there are no IPS refutations of $\operatorname{cnf}\left(\operatorname{IPS}_{r e f}^{\operatorname{alg}}\left(w, d, \varphi_{t, k, d}^{\star}\right)\right)$, and in particular no such IPS refutations of size at most $\left|\operatorname{cnf}\left(\operatorname{IPS}_{r e f}^{\text {alg }}\left(w, d, \varphi_{t, k, d}^{\star}\right)\right)\right|^{c_{0}}$ and degree at most $d(w)$ exist, whenever $w \geq|\gamma|^{c_{1}}$ and $t>k^{c_{1}}$, concluding the theorem. 
Remark 3.24. 1. Our lower bound in Conclusion eq. 16 and eq. 17 holds for all $t>k^{c_{1}}$. Note that as $t>k^{c_{1}}$ becomes bigger refuting $\operatorname{IPS}_{r e f}\left(t, d, \varphi_{r, m, d}^{\mathrm{cnf}}\right)$ becomes even harder, hence if there are no IPS refutations of $\operatorname{IPS}_{r e f}\left(t, d, \varphi_{r, m, d}^{\mathrm{cnf}}\right)$ of size $|\lambda|^{c_{0}}$ then there are no such refutations also for bigger t's.

2. Our lower bound in Conclusion eq. 16 and eq. 17 holds for all $m^{c_{1}}<r<m^{c_{2}}$. Note that as $r>m^{c_{1}}$ becomes bigger it becomes harder to refute $\varphi_{r, m, d}$ (as this would establish a stronger lower bound). Hence, the lower bound against $\operatorname{IPS}_{r e f}\left(t, d, \varphi_{r, m, d}^{\mathrm{cnf}}\right)$ becomes easier to prove. On the other hand, once $r>m^{c_{1}}$ becomes too big, namely exceeds $m^{c_{2}}$, $\varphi_{r, m, d}$ may become satisfiable, hence, refuting $\operatorname{IPS}_{r e f}\left(t, d, \varphi_{r, m, d}^{\mathrm{cnf}}\right)$ becomes easier: since $\varphi_{r, m, d}^{\mathrm{cnf}}$ has a satisfying assignment, we can use this assignment to refute the existence of a refutation of $\varphi_{r, m, d}^{\mathrm{cnf}}$.

It remains to construct an $\operatorname{IPS}^{\text {alg }}$ derivation of $\operatorname{IPS}_{r e f}^{\text {alg }}\left(t, d, \varphi_{r, m, d}^{\text {ecnf }}\right)$ from $\varphi_{t, k, d}^{\star}$ of size at most $\left|\varphi_{t, k, d}^{\star}\right|^{c_{3}}:$

Lemma 3.25. There is a constant $c_{3}$ such that if $\varphi_{r, m, d}$ is unsatisfiable, then under the above notation and parameters:

$$
\overbrace{\varphi_{t, k, d}^{\star}}^{\gamma} \frac{\left.|| \gamma\right|^{c_{3}, d(|\gamma|)}}{\operatorname{IPS} \operatorname{clg}} \operatorname{cnf}\left(\operatorname{IPS}_{r e f}^{\text {alg }}(t, \overbrace{\varphi_{r, m, d}^{\text {ecnf }}}^{\sigma})\right) .
$$

Proof: We start with $\gamma=\varphi_{t, k, d}^{\star}$ and recall that it is the extended CNF encoding (with some small size of added extension axioms that we will specify later in the proof of Prop. 3.26) of the equations "VNP $=\mathrm{VP}(t, k, d(t))$ " expressing that there exists a circuit of size $t$ and syntactic-degree $d(t)$ computing the permanent of dimension $k$ over $\mathbb{F}_{q}$.

The following is the crux of the argument: since the permanent polynomial perm $(\bar{x})$ of dimension $k$ is complete for VNP with $k / 6=\ell+N^{\prime}$ variables [Val79b], and since every unsatisfiable CNF has an IPS refutation whose certificate is computable in VNP by [GP18], and similarly every extended CNF formula has an IPS ${ }^{\text {alg }}$ refutation that is computable in VNP by Corollary 3.8, there exists an assignment $\bar{a}$ of field $\mathbb{F}_{q}$ elements and $\bar{x}$ variables to $\bar{x}$, with $\bar{x}$ having $k$ number of variables, such that $\operatorname{perm}\left(\bar{x}\lceil\bar{a})\right.$ is the IPS ${ }^{\text {alg }}$ refutation of the extended CNF $\varphi_{r, m, d}^{\text {ecnf }}$ (here, it will be sufficient to consider a refutation of $\varphi_{r, m, d}^{\mathrm{ecnf}}$ instead of $\varphi_{r, m, d}^{\star}$ ). Formally we have the following (note that any IPS $^{\text {alg }}$ derivation is also an IPS derivation, hence the formalisation is clearly doable also in IPS):

Proposition 3.26 (Grochow-Pitassi formalization in IPS ${ }^{\text {alg }}$ ). There is an IPS ${ }^{\text {alg }}$ derivation from $\varphi_{t, k, d}^{\star}$, denoted $\gamma$, of $\operatorname{IPS}_{r e f}^{\mathrm{alg}}\left(t, d, \varphi_{r, m, d}^{\mathrm{ecnf}}\right)$, with size at most $|\gamma|^{b}$, for some constant $b$.

Proof of Prop. 3.26. Consider $\varphi_{t, k, d}^{\star}$ which is defined to be the extended CNF of "VNP $=\mathrm{VP}(t, k, d(t))$ " (written as a set of corresponding SLP equations over $\mathbb{F}_{q}$ ) together with the following additional extension axioms in new variables (that are needed to invoke Prop. 3.6 in what follows):

extension axioms: for every circuit of the form $\operatorname{Coeff}_{M_{i}}(U(\bar{x}, \bar{w}))$ from eq. 9 in Definition 3.16 corresponding to "VNP $=\operatorname{VP}(r, m, d(r))$ " (note this is a smaller instance than "VNP $=\mathrm{VP}(t, k, d(t))$ " - for the latter we already have the corresponding SLP equations in $\varphi_{t, k, d}^{\star}$; note also that the variables in these two instances are disjoint) we have the following two:

1. the corresponding SLPs equations between extension variables for the gates in the circuit; 
2. the algebraic extension axioms (Definition 3.1) for every gate in the circuit.

The size of $\varphi_{t, k, d}^{\star}=\gamma$ is $t^{4} \cdot 2^{O\left(k^{2}+d\right)}$ by eq. 10. It is worth recalling that all our proofs use only the $\bar{w}$-variables because every polynomial in the $\bar{x}$-variables will eventually be fed into a Coeff $M(\cdot)$ operator for $M$ an $\bar{x}$-monomial, and this operator outputs $\bar{w}$-variables polynomials.

First, use Prop. 3.6 to derive from the extended $\mathrm{CNF} \varphi_{t, k, d}^{\star}$ the corresponding circuit equations "VNP $=\operatorname{VP}(t, k, d(t)) "$. The latter is formulated as a set of circuit equations expressing that the coefficients of the monomials of a universal circuit of size $t$ are the coefficients of perm $(\bar{x})$ of dimension $k$ (see Definition 3.16).

Consider $\bar{a}: \bar{x} \rightarrow \bar{x} \cup \mathbb{F}_{q}$ to be the assignment of variables and field elements to the $k \bar{x}$-variables that results in $\operatorname{perm}(\bar{x} \uparrow \bar{a})$ being a (correct) $\operatorname{IPS}^{\text {alg }}$ refutation of $\varphi_{r, m, d}^{\text {ecnf }}$ (such an assignment $\bar{a}$ exists as discussed above). It is left to efficiently derive in $\operatorname{IPS}^{\text {alg }}$ from "VNP $=\mathrm{VP}(t, k, d(t))$ " the equations of $\operatorname{IPS}_{r e f}^{\text {alg }}\left(t, d, \varphi_{r, m, d}^{\mathrm{ecnf}}\right)$. The latter is formulated by Definition 3.18 as a set of circuit equations expressing that the coefficients of the monomials of a universal circuit of size $s$ are precisely the coefficients of a polynomial that constitutes an IPS refutation of the extended CNF $\varphi_{r, m, d}^{\mathrm{ecnf}}$.

The idea is to use the equations of the form $\operatorname{Coeff}_{M_{i}}(U(\bar{x}, \bar{w}))=b_{i}$ from eq. 9 in "VNP $=\operatorname{VP}(t, k, d(t)) "$, and derive the equations that express that the coefficients of perm $(\bar{x}\lceil\bar{a})$ are precisely those of a polynomial that constitutes an IPS ${ }^{\text {alg }}$ refutation of the extended CNF $\varphi_{r, m, d}^{\mathrm{ecnf}}$. Since we know that $\operatorname{perm}\left(\bar{x}\lceil\bar{a})\right.$ is a correct IPS ${ }^{\text {alg }}$ refutation of $\varphi_{r, m, d}^{\mathrm{ecnf}}$, the equations for all coefficients will satisfy the conditions of Definition 3.18. Note that we will use three different assignments: first, the assignment $\bar{a}$ that will make perm $(\bar{x} \uparrow \bar{a})$ an IPS ${ }^{\text {alg }}$ refutation in the variables $\bar{x}$. We then compose separately $\bar{a}$ with two different assignments: the assignment of all zeros to those $\bar{x}$-variables that are identified as the IPS "placeholder" variables (denoted $\bar{y}$ ), and the assignment of the clauses of $\varphi_{r, m, d}^{\text {ecnf }}$ to these placeholder variables. The size bound on the IPS ${ }^{\text {alg }}$ proofs follows from the construction, which concludes Prop. 3.26 (for some constant $\ell$ ).

We first assert some polynomial identities. The following are polynomial identities, where $i$ ranges over $i \in[N]$ so that $\left\{M_{i}\right\}_{i=1}^{N}$ are the set of all possible $\bar{x}$-monomials of degree at most $d$ (as in Definition 3.16):

$$
\begin{aligned}
U(\bar{x} \uparrow \bar{a}, \bar{w}) & =\sum_{i \in[N]} \operatorname{Coeff}_{M_{i}}(U(\bar{x} \uparrow \bar{a}, \bar{w})) \cdot M_{i} \\
& =\left(\sum_{i \in[N]} \operatorname{Coeff}_{M_{i}}(U(\bar{x}, \bar{w})) \cdot M_{i}\right) \uparrow \bar{a} \\
& =\sum_{i \in[N]} \operatorname{Coeff}_{M_{i}}(U(\bar{x}, \bar{w})) \cdot\left(M_{i} \uparrow \bar{a}\right) .
\end{aligned}
$$

Equation eq. 22 holds by Definition 3.11 and Prop. 3.12, and eq. 23 holds because if we assign to $U$ and represent it as a sum of monomials or else we represent $U$ as a sum of monomials and then assign to it, we get the same polynomial. And eq. 24 is immediate because by construction Coeff $_{M_{i}}\left(U(\bar{x}, \bar{w})\right.$ ) (for some $\bar{x}$-monomial $M_{i}$ ) is a circuit that contains only the $\bar{w}$-variables. By eq. 24 we have the following polynomial identity for every $\bar{x}$-monomial $M$ :

$$
\operatorname{Coeff}_{M}\left(U(\bar{x}\lceil\bar{a}, \bar{w}))=\operatorname{Coeff}_{M}\left(\sum_{i \in[N]} \operatorname{Coeff}_{M_{i}}(U(\bar{x}, \bar{w})) \cdot\left(M_{i}\lceil\bar{a})\right)\right)\right.
$$

Since $\bar{b}$ is the coefficient vector of the permanent polynomial, we have also the following poly- 
nomial identity:

$$
\sum_{i \in[N]} b_{i} \cdot\left(M_{i} \uparrow \bar{a}\right)=\operatorname{perm}(\bar{x} \uparrow \bar{a})
$$

Let $M$ be an $\bar{x}$-monomial. By Corollary 3.14 and the fact that $\operatorname{Coeff}_{M_{i}}(U(\bar{x}, \bar{w}))$ (for some $\bar{x}$ monomial $\left.M_{i}\right)$ contains only the $\bar{w}$-variables, $\operatorname{Coeff}_{M}\left(\operatorname{Coeff}_{M_{i}}(U(\bar{x}, \bar{w}))\right)=\operatorname{Coeff}_{M_{i}}(U(\bar{x}, \bar{w}))$ which by the construction of $\operatorname{Coeff}_{M}(\cdot)$ implies the following polynomial identity:

$$
\left.\operatorname{Coeff}_{M}\left(\sum_{i \in[N]} \operatorname{Coeff}_{M_{i}}(U(\bar{x}, \bar{w})) \cdot\left(M_{i} \uparrow \bar{a}\right)\right)\right)=\sum_{i \in[N]} \operatorname{Coeff}_{M_{i}}(U(\bar{x}, \bar{w})) \cdot \operatorname{Coeff}_{M}\left(M_{i}\lceil\bar{a})\right) .
$$

By Fact 2.6 polynomial identities are proved in IPS (and similarly in IPS ${ }^{\text {alg }}$ ) with a size that is equivalent to the size of the circuits appearing in the equations. Therefore, in IPS ${ }^{\text {alg }}$ we can prove eq. 27 and then use substitutions of $\operatorname{Coeff}_{M_{i}}(U(\bar{x}, \bar{w}))$ for all $i$, by $b_{i}$, using the axioms $\operatorname{Coeff}_{M_{i}}(U(\bar{x}, \bar{w}))=b_{i}$. Thus, from eq. 25 , eq. 26 and eq. 27 we get in IPS ${ }^{\text {alg }}$

$$
\operatorname{Coeff}_{M}\left(U(\bar{x}\lceil\bar{a}, \bar{w}))=\operatorname{Coeff}_{M}\left(\sum_{i \in[N]} b_{i} \cdot\left(M_{i} \uparrow \bar{a}\right)\right)=\operatorname{Coeff}_{M}(\operatorname{perm}(\bar{x}\lceil\bar{a})),\right.
$$

where the left equation is by substitutions in IPS, and the right equation is a polynomial identity.

By assumption, $\bar{a}$ was chosen such that $\operatorname{perm}(\bar{x} \uparrow \bar{a})$ is an IPS ${ }^{\text {alg }}$ refutation of $\varphi_{r, m, d}^{\text {ecnf }}$. This in particular means that for the sake of clarity we can distinguish in $\bar{x} \uparrow \bar{a}$ two sets of variables: the "placeholder" variables (denoted $\bar{y}$ in Definition 2.2) and the "original" variables (denoted $\bar{x}$ in Definition 2.2). We do not need to use the placeholder variables $\bar{z}$ as in Definition 2.2 because we use IPS $^{\text {alg }}$ which does not use the boolean axioms (though some boolean axioms appear independently in $\left.\varphi_{r, m, d}^{\text {ecnf }}\right)$. Let us partition $\bar{x}$ into $\bar{x}^{\prime} \uplus \bar{y}$ whereas we denote those $\bar{x}$-variables in $\operatorname{perm}(\bar{x}\lceil\bar{a})$ that correspond to the placeholder variables by $\bar{y}$, and all the rest of the $\bar{x}$-variables in perm $(\bar{x}\lceil\bar{a})$ (and similarly in perm $(\bar{x})$ and in $U(\bar{x}, \bar{w}))$ by $\bar{x}^{\prime}$. Note that as an $\operatorname{IPS}^{\text {alg }}$ refutation, $\operatorname{perm}(\bar{x} \uparrow \bar{a})$, or $\operatorname{perm}\left(\bar{x}^{\prime} \uparrow \bar{a}, \bar{y} \uparrow \bar{a}\right)$ in the new notation, must have all placeholder variables $\bar{y}$ unassigned, hence $\bar{a}$ leaves $\bar{y}$ intact, meaning that $\operatorname{perm}\left(\bar{x}^{\prime}\lceil\bar{a}, \bar{y} \uparrow \bar{a})=\operatorname{perm}\left(\bar{x}^{\prime} \uparrow \bar{a}, \bar{y}\right)\right.$ is the IPS alg refutation of $\varphi_{r, m, d}^{\mathrm{ecnf}}$.

Denote by $\uparrow \bar{y}=\mathbf{0}$ the restriction that assigns 0 to all $\bar{y}$ variables, and by $\uparrow \bar{y}=\varphi_{r, m, d}^{\mathrm{ecnf}}$ the restriction that assigns to each variable $y_{i}$ the corresponding $i$ th equation from $\varphi_{r, m, d}^{\text {ecnf }}$ (equation $f=g$ here means the term $f-g$ ). Denote by $\lceil\bar{a} \cup \bar{y}=\mathbf{0}$ the restriction that first assigns $\bar{a}$ to the $\bar{x}^{\prime}$-variables and then assigns zeros to the $\bar{y}$-variables (since $\bar{x}^{\prime}$ and $\bar{y}$ are disjoint the order of assignments is unimportant). Similarly, denote by $\uparrow \bar{a} \cup \bar{y}=\varphi_{r, m, d}^{\text {ecnf }}$ the restriction that first assigns $\bar{a}$ to the $\bar{x}^{\prime}$-variables and then assigns to each variable $y_{i}$ the corresponding $i$ th equation from $\varphi_{r, m, d}^{\text {ecnf }}$.

In the same way we proved eq. 28 in IPS ${ }^{\text {alg }}$ using the assignment $\bar{a}$ we can prove with a linear size proof the following, for every $\bar{x}$-monomial $M$ and for the assignment $\bar{a} \cup \bar{y}=\mathbf{0}$ :

$$
\begin{array}{rlrl}
\operatorname{Coeff}_{M}(U(\bar{x} \uparrow \bar{a} \cup \bar{y}=\mathbf{0}, \bar{w})) & =\operatorname{Coeff}_{M}\left(U\left(\bar{x}^{\prime} \uparrow \bar{a}, \mathbf{0}, \bar{w}\right)\right) & \quad\left(\text { by } \bar{x}=\bar{x}^{\prime} \uplus \bar{y}\right) \\
& =\operatorname{Coeff}_{M}\left(\sum_{i \in[N]} b_{i} \cdot\left(M_{i} \uparrow \bar{a} \cup \bar{y}=\mathbf{0}\right)\right) \\
& =\operatorname{Coeff}_{M}(\operatorname{perm}(\bar{x} \uparrow \bar{a} \cup \bar{y}=\mathbf{0})) \\
& =\operatorname{Coeff}_{M}\left(\operatorname{perm}\left(\bar{x}^{\prime} \uparrow \bar{a}, \bar{y} \uparrow \mathbf{0}\right)\right)=0, & \text { (similar to eq. 28) }
\end{array}
$$


where eq. 29 holds because by assumption perm $\left(\bar{x}\lceil\bar{a})\right.$ is a correct IPS ${ }^{\text {alg }}$ refutation of $\varphi_{r, m, d}^{\text {ecnf }}$ which means that every nonzero monomial in it is a product of some placeholder $\bar{y}$-variables, and we assign zeros to the $\bar{y}$-variables. In particular eq. 29 holds for every $M$ monomial in the $\bar{x}^{\prime}$-variables, which are precisely the desired first set of equations in $\operatorname{IPS}_{r e f}^{\text {alg }}\left(t, d, \varphi_{r, m, d}^{\text {ecnf }}\right)$ (corresponding to eq. 11 in Definition 3.18).

Similarly, we can consider the assignment $\bar{a} \cup \bar{y}=\varphi_{r, m, d}^{\mathrm{ecnf}}$. Here we use the fact that $\varphi_{t, k, d}^{\star}$ provides all the extension axioms for gates in the circuits of $\varphi_{r, m, d}^{\text {ecnf }}$, so that we can write $\varphi_{r, m, d}^{\text {ecnf }}$ with SLPs (note that $\varphi_{r, m, d}^{\mathrm{ecnf}}$ is a set of polynomials in the $\bar{x}^{\prime}$-variables). Hence, similarly as above, we get a proof in IPS ${ }^{\text {alg }}$ of:

$$
\begin{aligned}
\operatorname{Coeff}_{M}\left(U\left(\bar{x}\left\lceil\bar{a} \cup \bar{y}=\varphi_{r, m, d}^{\mathrm{enf}}, \bar{w}\right)\right)\right. & =\operatorname{Coeff}_{M}\left(\operatorname{perm}\left(\bar{x}^{\prime} \uparrow \bar{a}, \bar{y} \uparrow \varphi_{r, m, d}^{\mathrm{ecnf}}\right)\right) \\
& = \begin{cases}1, & M=1 \quad \text { (i.e., the constant } 1 \text { monomial }) ; \\
0, & \text { all other } \bar{x}^{\prime} \text {-monomials } M .\end{cases}
\end{aligned}
$$

It remains to inspect that the size of all the circuits in the IPS ${ }^{\text {alg }}$ proofs above are bounded by a polynomial in $\gamma=\left|\varphi_{t, k, d}^{\star}\right|=t^{4} \cdot 2^{O\left(k^{2}+d\right)}$. For this notice that when we apply Coeff $M(\cdot)$ with $M$ of degree $d$ on a polynomial of size $t^{4} \cdot 2^{O\left(k^{2}+d\right)}$ as in eq. 27 we get by Prop. 3.12 a size of $7^{d} \cdot t^{4} \cdot 2^{O\left(k^{2}+d\right)}=t^{4} \cdot 2^{O\left(k^{2}+d\right)}$.

We are now in a position to complete the proof of Lemma 3.25:

$$
\varphi_{t, k, d}^{\star} \frac{\left.|| \gamma\right|^{\ell}, d(|\gamma|)}{\operatorname{IPS}^{\mathrm{alg}}} \operatorname{IPS}_{r e f}^{\mathrm{alg}}\left(t, d, \varphi_{r, m, d}^{\mathrm{ecnf}}\right) \quad \text { (by Prop. 3.26), }
$$

and by using again Prop. 3.6 (in the other direction to that in Prop. 3.26) and the fact that we have extension axioms for all the subcircuits in $\operatorname{IPS}_{r e f}^{\text {alg }}\left(t, d, \varphi_{r, m, d}^{\text {ecnf }}\right.$ ) by definition of $\varphi_{t, k, d}^{\star}$ (this is where we use the fact that we started with $\varphi_{t, k, d}^{\star}$ and not merely $\left.\varphi_{t, k, d}\right)$, to get

$$
\frac{|\gamma|^{\ell^{\prime}, d(|\gamma|)}}{\operatorname{IPS}^{\text {alg }}} \operatorname{ecnf}\left(\operatorname{IPS}_{r e f}^{\mathrm{alg}}\left(t, d, \varphi_{r, m, d}^{\mathrm{ecnf}}\right)\right)
$$

for some constant $\ell^{\prime}$.

Since $\operatorname{cnf}(C=0) \subset \operatorname{ecnf}(C=0)$ for every (set of) circuits $C$, we get an IPS ${ }^{\text {alg }}$ derivation from the right hand side of eq. 32 of $\operatorname{cnf}\left(\operatorname{IPS}_{r e f}^{\text {alg }}\left(t, d, \varphi_{r, m, d}^{\text {ecnf }}\right)\right)$. Overall, we got a derivation from $\varphi_{t, k, d}^{\star}$ of $\operatorname{cnf}\left(\operatorname{IPS}_{r e f}^{\mathrm{alg}}\left(t, d, \varphi_{r, m, d}^{\mathrm{enf}}\right)\right)$ with size at most $\left|\gamma^{c_{3}}\right|$ for some constant $c_{3} \geq \ell+\ell^{\prime}$.

This concludes Lemma 3.25.

This concludes the proof of the $(\Rightarrow)$ direction of Thm. 3.22.

\subsubsection{The interesting range of parameters}

Let us now discuss different ranges of parameters and what they mean for Thm. 3.22. In Remark 3.19 we have already mentioned that the degree of IPS refutations we work with should not be too big, as otherwise, although the argument is correct, it is trivial since there is always a short refutation.

Similarly, it is important for us that the size of our formulas is small enough. The reason is once more that otherwise we get to the following uninteresting case: the size of the formula $\operatorname{cnf}\left(\operatorname{IPS}_{r e f}\left(t, d, \varphi_{r, m, d}^{\mathrm{cnf}}\right)\right)$ is exponential in the number of its variables, hence it must be easy to 
refute. Thus, if it is satisfiable it has no IPS refutation by soundness of IPS, and if it is unsatisfiable it has an IPS refutation of size polynomial in its size; this bring us to Part 2 in the dichotomy conjecture Thm. 4.5: the hard candidates alternate between being satisfiable and hence having no short refutations by soundness, and being unsatisfiable but having a short refutation trivially.

More precisely, we distinguish between two types of exponential growth functions: good and bad, as follows. By Sect. 3.1.3, the size of "VNP $=\mathrm{VP}(t, n, d)$ " is $t^{4} \cdot 2^{O\left(n^{2}+d\right)}$ with $t$ the number of variables. Whenever $t \geq\left(n^{2}+d\right)^{c}$, for some constant $c>1$, the term $t^{4} \cdot 2^{O\left(n^{2}+d\right)}$ is considered a good exponential because it is exponentially smaller than $2^{t}$, and the latter term is considered a bad exponential (recall that the degree $d \leq t^{\epsilon}$ for some $0<\epsilon<1$, hence $t=\left(n^{2}+d\right)^{c}$ is a valid equality).

Corollary 3.27. Thm. 3.22 is interesting whenever $t \geq\left(n^{2}+d(t)\right)^{c}$ (and when we assume that $d(t) \leq 2^{\epsilon}$ for some $0<\epsilon<1$ ), in the sense that in this range of parameters the hard candidate $\operatorname{cnf}\left(\operatorname{IPS}_{r e f}\left(t, d, \varphi_{r, m, d}^{\mathrm{cnf}}\right)\right)$ is hypothesized to be both unsatisfiable and for which no polynomial-size IPS refutations exist.

We also have the following immediate corollary:

Corollary 3.28. Thm. 3.22 holds for any proof system that simulates IPS.

\section{Candidate Hard Formulas for Every Propositional Proof System}

While the previous section dealt with algebraic proof systems and used the specific properties of the IPS refutation systems, in this section we consider similar notions of diagonalisations for general propositional proof systems.

\subsection{Iterated Lower Bound Formulas}

For basic definitions on propositional proof complexity, please refer to the comprehensive overview of Krajicek [Kra19].

Definition 4.1. Given propositional proof system $R$, propositional formula $\phi$ and size function $s: \mathbb{N} \rightarrow \mathbb{N}, \mathrm{Ib}_{R}(\lceil\phi\rceil, s)$ is a propositional DNF formula of size poly $(|\phi|+s(|\phi|))$ over poly $(|\phi|+s(|\phi|))$ variables expressing that there is no $R$-proof of $\phi$ having size $s(|\phi|)$.

More explicitly, the formula $\mathrm{Ib}_{R}(\phi, s)$ contains $s$ variables $y_{1}, \ldots, y_{s}$ encoding $R$-proofs of length $s$ and poly $(|\phi|+s)$ auxiliary variables encoding the computation of the relation $R^{14}$, to verify that $y_{1}, \ldots, y_{s}$ does not constitute an $R$ proof of $\phi$. For a detailed description of how this encoding works, see [Pud20].

Definition 4.2 (Reasonably Strong Proof System). We say that a propositional proof system $R$ is reasonably strong if it satisfies the following conditions:

1. $R$ p-simulates Res (Resolution).

2. $R$ is closed under partial assignments, i.e., if there are polynomial-size $R$-proofs of $\phi$ and $\bar{a}$ is a partial truth assignment to the variables of $\phi$, then there are polynomial-size $R$-proofs of $\phi(\bar{a})$.

\footnotetext{
${ }^{14} \mathrm{~A}$ propositional proof system $P$ can be interpreted as relation $R$ where $R(x, y)$ hold iff $y$ is a $P$-proof of the tautology encoded by $x$.
} 
3. $R$ has poly-size proofs of its own reflection principle $\mathrm{Ib}_{R}(\phi, s) \vee \phi$.

4. $R$ is closed under modus ponens, i.e., if there are polynomial-size $R$-proofs of $\tau \vee \neg \phi$ and of $\phi \vee \psi$ for DNFs $\tau, \phi, \psi$, then there are polynomial-size $R$-proofs of $\tau \vee \psi$.

The conditions above are satisfied for any standard strong enough propositional proof system, eg., Frege and Extended Frege.

We now formally define iterated lower bound formulas relative to a propositional proof system $R$.

Definition 4.3 (Iterated Lower Bound Formulas). Given propositional proof system R, propositional formula $\phi$ and size function $s: \mathbb{N} \rightarrow \mathbb{N}$, the sequence $\left\{\mathrm{Ib}_{R}^{k}(\phi, s)\right\}, k=0 \ldots \infty$ is defined inductively as follows:

1. $\operatorname{lb}_{R}^{0}(\phi, s)=\phi$

2. $\mathrm{Ib}_{R}^{k+1}(\phi, s)=\mathrm{Ib}_{R}\left(\mathrm{Ib}_{R}^{k}(\phi, s), s\right)$.

The meaning of the iterated lower bound formulas is thus that we consider a single fixed base hard formula $\phi$, and we iterate the statement that expresses the lower bound against this base hard formula.

Lemma 4.4. Let $R$ be any reasonably strong propositional proof system, and let $\tau$ be a nontautology. Then there are $R$-proofs of $\mathrm{Ib}_{R}(\tau, s)$ of size poly $\left(\left|\mathrm{Ib}_{R}(\tau, s)\right|\right)$.

Proof: Since $\tau$ is a non-tautology, there is an assignment $\bar{a}$ to the variables of $\tau$ such that $\tau(\bar{a})$ evaluates to false. Since $R$ is reasonably strong, it simulates Res and hence can prove $\neg \tau(\bar{a})$ by substituting the assignment $\bar{a}$ into $\tau$ Since $R$ is reasonably strong, $R$ can prove its own reflection principle efficiently, and since $R$ is closed under partial assignments, it can also prove $\mathrm{lb}_{R}(\tau, s) \vee \tau(\bar{a})$, using the fact that the variables of $\tau$ do not occur in $\mathrm{lb}_{R}(\tau, s)$. It follows from the closure under modus ponens that $R$ can prove $\mathrm{Ib}_{R}(\phi, s)$ efficiently.

We show a dichotomy for iterated lower bound formulas: either they are all hard (and hence all tautologies), or they divide up according to the parity of $k$, with one parity corresponding to nontautologies and the other parity corresponding to tautologies with short proofs. This dichotomy shows there are only two extreme cases - the highly interesting and the trivial one, with nothing in between: either the iterated lower bound formulas are all hard proof complexity instances, or none of them is (since when they do not have short proofs it follows from them have no proof at all).

Theorem 4.5 (Dichotomy for Iterated Lower Bound Formulas). Let $R$ be a reasonably strong propositional proof system and $s: \mathbb{N} \rightarrow \mathbb{N}$. There is a constant $c$ such that for every $s$ with $s(n)>n^{c}$ for all $n \in \mathbb{N}$ and for every $\phi$ that does not have $R$-proofs of size $s(|\phi|)$, exactly one of the following holds:

1. For every integer $k \geq 0, \phi_{k}=\mathrm{Ib}_{R}^{k}(\phi, s)$ is a tautology that does not have $R$-proofs of size $s\left(\left|\phi_{k}\right|\right)$.

2. There is an integer $k \geq 0$ such that for every integer $i \geq 0, \phi_{k+i}$ is not a tautology (and hence does not have $R$-proofs of any size) if $i$ is odd, and $\phi_{k+i}$ is a tautology with $R$-proofs of size at most poly $\left(s\left(\left|\phi_{k+i}\right|\right)\right)$ if $i$ is even. 
Proof: Suppose that for every integer $k \geq 0, \phi_{k}$ is a tautology. We show that this implies that $\phi_{k}$ is also hard in the sense that it does not have $R$-proofs of size $s\left(\left|\phi_{k}\right|\right)$. Indeed, since $\phi_{j}$ is a tautology for every $j$, it follows that $\phi_{k+1}$ is a tautology. Since $\phi_{k+1}$ asserts that $\phi_{k}$ does not have $R$-proofs of size $s\left(\left|\phi_{k}\right|\right)$, it follows that $\phi_{k}$ is indeed hard as claimed. Thus, in this case, the first item in the statement of the theorem holds.

Otherwise, since $\phi_{0}=\phi$ is a tautology with no $R$-proofs of size $s(|\phi|)$, there is a least positive integer $k$ such that $\phi_{k}$ is a tautology but $\phi_{k+1}$ is a non-tautology. Since $\phi_{k+1}$ asserts that $\phi_{k}$ does not have $R$-proofs of size $s\left(\left|\phi_{k}\right|\right)$, it follows that $\phi_{k}$ does indeed have $R$-proofs of size $s\left(\left|\phi_{k}\right|\right)$. We will show by induction in this case that for each integer $i \geq 0$ the following statement $S(i)$ holds: $\phi_{k+2 i+1}$ is not a tautology (and hence does not have $R$-proofs of any size), and $\phi_{k+2 i}$ is a tautology with $R$-proofs of size at most $s\left(\left|\phi_{k+2 i}\right|\right)$.

We first establish the base case $S(0)$. When $i=0$, by assumption on $\phi_{k}$, we have that $\phi_{k+2 i}=\phi_{k}$ is a tautology with $R$-proofs of size at most $s\left(\left|\phi_{k}\right|\right)$. Also, by assumption on $k, \phi_{k+1}$ is a nontautology, and since $R$ is sound, it follows that $\phi_{k+1}$ does not have $R$-proofs of any size.

For the inductive step, we assume that $S(i)$ has been shown and deduce $S(i+1)$. Since $S(i)$ is true, we have that $\phi_{k+2 i}$ is a tautology with $R$-proofs of size at most $s\left(\left|\phi_{k+2 i}\right|\right)$ and $\phi_{k+2 i+1}$ is not a tautology (and hence does not have $R$-proofs of any size). We have that $\phi_{k+2 i+2}$ asserts that $\phi_{k+2 i+1}$ does not have $R$-proofs of size $s\left(\left|\phi_{k+2 i+1}\right|\right)$, which is tautologous since $\phi_{k+2 i+1}$ does not have $R$-proofs of any size. In order to show that $\phi_{k+2 i+2}$ has $R$-proofs of size at most $s\left(\left|\phi_{k+2 i+2}\right|\right)$, we simply apply Lemma 4.4 with $\tau=\phi_{k+2 i+1}$, where $c$ is a constant such that $\operatorname{lb}_{R}(\tau, s)$ has $R$-proofs of size at most $\left|\mathrm{lb}_{R}(\tau, s)\right|^{c}$. Since $s(n)>n^{c}$ for all $n \in \mathbb{N}$, we have that $\mathrm{Ib}_{R}(\tau, s)$ has $R$-proofs of size at most $s\left(\left|\mathrm{Ib}_{R}(\tau, s)\right|\right)$. It follows that $\phi_{k+2 i+3}$ is not a tautology, since $\phi_{k+2 i+3}$ asserts that $\phi_{k+2 i+2}$ does not have $R$-proofs of size at most $s\left(\left|\phi_{k+2 i+2}\right|\right)$, which is false by the previous line, and we are done.

We call the first item of Thm. 4.5 the useful case of the dichotomy, as this is the case that gives us hard tautologies for $R$.

Iterated Lower Bound Hypothesis: Let $R$ be a reasonably strong propositional proof system that is not polynomially bounded. Then there is a super-polynomial function $s: \mathbb{N} \rightarrow \mathbb{N}$ and a formula $\phi$ with no $R$-proofs of size $s(|\phi|)$ such that for all non-negative integers $k, \phi_{k}=\mathrm{lb}_{R}^{k}(\phi, s)$ is a tautology that does not have $R$-proofs of size $s\left(\left|\phi_{k}\right|\right)$.

We observe that the Iterated Lower Bounds Hypothesis fails if optimal proof systems exist. The existence of optimal proof systems is not widely believed; indeed, under this assumption, there is a complexity collapse.

Proposition 4.6. Suppose there exists an optimal proof system $R$ that is reasonably strong. Then for any time-constructible super-polynomial function $s: \mathbb{N} \rightarrow \mathbb{N}$ and any formula $\phi$, there is a positive integer $k$ such that $\phi_{k}=\mathrm{Ib}_{R}^{k}(\phi, s)$ is not a tautology.

Proof: Suppose there exists an optimal proof system $R$ that is reasonably strong. Assume, for the sake of contradiction, that there is a formula $\phi$ and a time-constructible super-polynomial function $s$ such that $\phi_{k}=\mathrm{Ib}_{R}^{k}(\phi, s)$ is a tautology for every positive integer $k$. Define the propositional proof system $R^{\prime}$ which is $R$ plus the formulas $\phi_{k}$ added as axioms. Thus $R^{\prime}$ has polynomial-size (indeed size zero) proofs of $\phi_{k}$ for each $k$. $R^{\prime}$ is indeed a propositional proof system: it is complete since $R$ is a propositional proof system, it is sound since the formulas $\phi_{k}$ are tautologies by assumption, and it is polynomially verifiable since $R$ is polynomially verifiable and the sequence $\phi_{k}$ is polynomial-time computable by time-constructibility of $s$.

Since $R$ is optimal, we have that $R$ polynomially simulates $R$ '. Since $R^{\prime}$ has constant-size proofs of each $\phi_{k}$, this means that there are $R$-proofs of $\phi_{k}$ of size at most $\left|\phi_{k}\right|^{c}$ for each $k$, where $c$ is a 
constant. Choose $k$ large enough so that $s\left(\left|\phi_{k}\right|\right)>\left|\phi_{k}\right|^{c}$. Since $\phi_{k+1}$ is a tautology, we have that $\phi_{k}$ does not have proofs of size $s\left(\left|\phi_{k}\right|\right)$ and hence does not have proofs of size $\left|\phi_{k}\right|^{c}$, which is a contradiction.

Recently, it was shown that Resolution is NP-hard to automate, by using a reduction based on proof complexity lower bound formulas [AM20, Gar19].

Theorem 4.7 (Atserias-Muller [AM20], Garlik [Gar19]). Let $s: \mathbb{N} \rightarrow \mathbb{N}$ be any super-polynomial function such that $s(n)=2^{n^{o(1)}}$. For any formula $\phi$, if $\phi$ is a tautology, then $\tau=\operatorname{lb}_{\operatorname{Res}}(\phi)$ does not have Res-proofs of size $s(|\tau|)$.

It follows by induction, since the base formula $\phi$ is hard, that the Iterated Lower Bounds Hypothesis holds at least for the relatively weak proof system Resolution:

Corollary 4.8. Let $s: \mathbb{N} \rightarrow \mathbb{N}$ be any super-polynomial function such that $s(n)=2^{n^{o(1)}}$ and let $\phi$ be a tautology with no Resolution proofs of size $s(|\phi|)$ (e.g., the Pigeonhole Principle). Then for all non-negative integers $k, \phi_{k}=\mathrm{Ib}_{\operatorname{Res}}^{k}(\phi, s)$ is a tautology that does not have $R$-proofs of size $s\left(\left|\phi_{k}\right|\right)$.

\subsection{Iteration Preserves Hardness for Random Truth Table Formulas}

The Iterated Lower Bounds hypothesis is about the hardness of a fixed propositional formula to which the proof complexity lower bound operator is applied an unbounded number of times. In this section, we give partial evidence in favour of the Hypothesis by considering a candidate hard distribution on formulas, and showing that under a reasonable hypothesis, applying the proof complexity lower bound operator a fixed number of times to a formula sampled from the distribution does not decrease hardness. The interesting aspect of this result is that the hardness holds for a sufficiently strong propositional proof system $R$ rather than a relatively weak one such as Resolution.

Definition 4.9 (Truth Table Formulas [PS19]). Given a boolean function $f_{n}$ on $n$ variables and a size parameter $t$, ttable $(f, t)$ is a propositional DNF formula of size $N=\tilde{O}\left(2^{n} s^{3}\right)$ over $\tilde{O}(s)$ variables expressing that $f$ does not have boolean circuits of size $s$. The distribution $\operatorname{Randtt}(n, t)$ over ttable $(f, t)$, where $f$ is chosen uniformly at random from all boolean functions on $n$ variables, is called the distribution of random truth table formulas.

Conjecture 4.10 (Rudich's Conjecture [Rud97]). There is a constant $\ell$ for which there is no sequence of polynomial-size non-deterministic circuits $\left\{C_{m}\right\}$ such that for infinitely many $m$ for which $m=2^{n}$ for some non-negative integer $n$ :

1. If $C_{m}$ accepts a string $y$, then $y$ is the truth table of a boolean function $f_{n}$ that does not have boolean circuits of size $n^{\ell}$.

2. $C_{m}$ accepts at least an inverse polynomial fraction of all inputs.

Definition 4.11 (Distributional Iterated Lower Bound Formulas). Let $\mathfrak{D}=\left\{D_{N}\right\}$ be a sequence of distributions, where $D_{N}$ is supported on propositional formulas of size $N$. Given propositional proof system $R$, non-negative integer $k$ and size function $s: \mathbb{N} \rightarrow \mathbb{N}$, the sequence of distributions $\mathrm{Ib}_{R}^{k}(\mathfrak{D}, s)$ is defined via induction on $k$ as follows:

1. $\operatorname{lb}_{R}^{0}(\mathfrak{D}, s)=\mathfrak{D} ;$

2. $\mathrm{Ib}_{R}^{k+1}(\mathfrak{D}, s)$ is the sequence of distributions on formulas $\mathrm{Ib}_{R}(\phi, s)$ where $\phi$ is sampled from $\mathrm{Ib}_{R}^{k}(\mathfrak{D}, s)$

The following theorem strengthens Lemma 3 in [PS19], which corresponds to the case $k=2$. 
Theorem 4.12. If Rudich's Conjecture holds, then there exist a propositional proof system $R$ efficiently simulating Extended Frege and a constant $\ell>0$ such that for every large enough $c>0$ and every non-negative integer $k, \mathrm{Ib}_{R}^{k}\left(D_{N}, N^{c}\right)$ is a tautology with no R-proofs of size $\left|\mathrm{Ib}_{R}^{k}\left(D_{N}, N^{c}\right)\right|^{c}$ with probability $1-o(1)$ for all large enough $N$, where $D_{N}=\operatorname{Randtt}\left(n, n^{\ell}\right)$ (for $N$ an appropriate function of $n$ and $\ell$ as given by Definition 4.9).

Proof: The proof is by induction on $k$. Let $\ell>0$ be the constant in Conjecture 4.10 and let $R$ be a propositional proof system and $c$ be a large enough constant to be specified later.

We will establish the case $k=0$ for every propositional proof system $R$, under Rudich's Conjecture. We have that $\mathrm{Ib}_{R}^{k}\left(D_{N}, N^{c}\right)=D_{N}$. Since a random boolean function on $n$ variables has circuits of size $n^{\ell}$ with exponentially small probability, a formula $\tau$ sampled according to $D_{N}$ is a tautology with high probability. Moreover, Rudich's Conjecture implies that $\tau$ does not have $R$-proofs (or indeed proofs in any propositional proof system) of size $N^{c}$ with high probability. To see this, note that $R$ with size bound $s$ defines a non-deterministic algorithm $A_{R}$ running in time $\operatorname{poly}(s)$ for the problem $\overline{\operatorname{MCSP}}\left[n^{\ell}\right]$ asking whether a given truth table $y$ of a boolean function $f$ on $n$ bits requires circuits of size greater than $n^{\ell}: A_{R}$ checks if ttable $\left(f, n^{\ell}\right)$ has $R$-proofs of size $s$. $A_{R}$ only accepts on hard boolean functions, by the soundness of $R$, satisfying the first item of Conjecture 4.10. If $A_{R}$ accepted $\tau$ for even a $1 / N$ fraction of truth-table tautologies $\tau$, this would contradict the second item of Conjecture 4.10.

We define $R$ to be the propositional proof system that is Extended Frege together with axioms stating that ttable $\left(f_{n}^{\text {SAT }}, 2 n^{\ell}\right)$ holds, where $f_{n}^{\text {SAT }}$ is the truth table of SAT on $n$ variables. The axioms indeed hold under Rudich's Conjecture, as Rudich's Conjecture implies that NP does not have polynomial-size circuits. It is not hard to verify that $R$ is reasonably strong according to Definition 4.2.

Now suppose we have established the assertion for all non-negative integers smaller than $k$ and would like to establish it for $k$. The inductive strategy builds partly on ideas in [PS19]. We have by the inductive assumption that with probability $1-o(1)$ for $\psi$ sampled from $D_{N}, \mathrm{lb}_{R}^{k-1}\left(\psi, N^{c}\right)$ does not have $R$-proofs of size $\left|\mathrm{lb}_{R}^{k-1}\left(\psi, N^{c}\right)\right|^{c}$. It follows immediately that with probability $1-o(1)$, for $\psi$ sampled from $D_{N}, \mathrm{lb}_{R}^{k}\left(\psi, N^{c}\right)$ is a tautology.

For the lower bound on $\mathrm{Ib}_{R}^{k}\left(D_{N}, N^{c}\right)$, we will treat $k$ differently depending on its parity.

Case 1: $k$ is even. We will show inductively that if $\psi_{k}=\mathrm{lb}_{R}^{k}\left(\psi, N^{c}\right)$ is a tautology, then so is $\psi$. Indeed, this is trivially true when $k=0$, and we show that if $\psi_{k}$ is a tautology, then so is $\psi_{k-2}$. Assume contrapositively that $\psi_{k-2}$ is not a tautology. This means that $\psi_{k-1}$ is a tautology with $R$-proofs of size $\left|\psi_{k-1}\right|^{c}$, since $R$ is reasonably strong, using Lemma 4.4, where $c$ is greater than the exponent of the polynomial in Lemma 4.4. But this implies $\psi_{k}$ is not a tautology, contradicting our assumption on $\psi$.

Now we use the assumption of Rudich's Conjecture to complete the inductive step. It remains to prove that with probability $1-o(1)$, for $\psi$ sampled from $D_{N}, \mathrm{Ib}_{R}^{k}\left(\psi, N^{c}\right)$ does not have $R$ proofs of size $\left|\mathrm{Ib}_{R}^{k}\left(\psi, N^{c}\right)\right|^{c}$. Suppose, for the sake of contradiction that with probability $\Omega(1)$, for infinitely many $N$, for $\psi$ sampled from $D_{N}, \mathrm{Ib}_{R}^{k}\left(\psi, N^{c}\right)$ has $R$-proofs of the desired size. We use this to define a non-deterministic polynomial-time algorithm that accepts a constant fraction of truth tables of hard boolean functions on $n$ bits and does not accept any easy boolean functions on $n$ bits, for infinitely many $n$, in contradiction to Rudich's Conjecture. Given a truth table of a boolean function $f_{n}$, the algorithm checks if there is an $R$-proof of $\psi_{k}=\mathrm{Ib}_{R}^{k}\left(\psi, N^{c}\right)$ of size at most $\left|\psi_{k}\right|^{c}$, where $\psi=\operatorname{ttable}\left(f_{n}, n^{\ell}\right)$, and accepts if yes. If the algorithm does accept on $\psi_{k}$, then by the soundness of $R, \psi_{k}$ is a tautology, and since $k$ is even, so is $\psi$ by the inductive argument in the previous paragraph. Hence $f$ is indeed a hard boolean function, as desired. Moreover, for $f_{n}$ chosen uniformly at random, the algorithm accepts with probability $\Omega(1)$ by assumption, for infinitely many $n$, contradicting Rudich's Conjecture. 
Case 2: $k$ is odd. We need a slightly more involved argument, which generalizes the argument used to show Lemma 2 in [PS19]. Since Rudich's Conjecture holds, it follows that there are succinct hitting sets against polynomial-size non-deterministic circuits, i.e., a sequence $\left\{H_{m}\right\}$ of sets of strings in $\{0,1\}^{m}$, where each string in $H_{m}$ is a truth table of a boolean function on $\log (m)$ inputs with circuits of size $\log (m)^{\ell}$ (we assume without loss of generality that $m$ is a power of 2 ), such that for every sequence $\left\{C_{m}\right\}$ of non-deterministic circuits that accept a $\Omega(1)$ fraction of their inputs, at least one element of $H_{m}$ is accepted by $C_{m}$ for all large enough $m$. Also, as mentioned before, since Rudich's Conjecture holds, it follows that SAT does not have polynomial-size circuits. Using a straightforward argument, the sequence $\left\{H_{m}^{\prime}\right\}$ of sets of strings in $\{0,1\}^{m}$, where each $H_{m}^{\prime}$ is $f_{\log (m)}^{\mathrm{SAT}} \oplus y$ for $y \in H_{m}$, is also a hitting set sequence against polynomial-size non-deterministic circuits, but in this case the sets consist of truth tables of hard boolean functions on $\log (m)$ inputs. We have that ttable $\left(z, n^{\ell}\right)$ is a tautology for $z \in H_{m}^{\prime}$ when $m=2^{n}$ is large enough. Moreover, by the same argument as in the proof of Lemma 2 in [PS19], for each $z \in H_{m}^{\prime}$, ttable $\left(z, n^{\ell}\right)$ has $R$-proofs of size at most $\left|\operatorname{ttable}\left(z, n^{\ell}\right)\right|^{c}$, using the fact that $R$ p-simulates Extended Frege and has ttable $\left(f_{n}^{\mathrm{SAT}}, 2 n^{\ell}\right)$ as an axiom.

These facts can be used to show by the inductive argument in the proof of Thm. 4.5 that the second item of Thm. 4.5 holds for the formulas $\psi_{k}=\mathrm{Ib}_{R}^{k}\left(\psi, N^{c}\right)$ where $\psi=\operatorname{ttable}\left(z, n^{\ell}\right)$ for $z \in H_{m}^{\prime}$ : they are tautologies with short $R$-proofs when $k$ is even, and non-tautologies (and hence without any $R$-proofs at all) when $k$ is odd. In the current case of our inductive step, $k$ is odd, and hence every such formula $\psi_{k}$ is a non-tautology, and hence does not have short proofs. Now suppose for the sake of contradiction, that with probability $\Omega(1)$ over uniformly chosen boolean function $f_{n}$ on $n$ variables, for infinitely many $n, \mathrm{lb}_{R}^{k}\left(\psi, N^{c}\right)$ has short $R$-proofs, where $\psi=\operatorname{ttable}\left(f_{n}, n^{\ell}\right)$. This means that the polynomial-time non-deterministic algorithm that on input $f_{n}$ checks if there is a short $R$-proof of $\mathrm{Ib}_{R}^{k}\left(\operatorname{ttable}\left(f_{n}, n^{\ell}\right), N^{c}\right)$ accepts a constant fraction of functions $f_{n}$. However, none of the functions $z$ for $z \in H_{m}^{\prime}$ is accepted. This contradicts the assumption that $\left\{H_{m}^{\prime}\right\}$ is a hitting set sequence for all $m$, and hence also contradicts Rudich's Conjecture.

\section{Acknowledgements}

We wish to thank Jan Pich for very helpful discussions during the work on this paper. We also thank Jan Krajíček for helpful comments on an earlier draft of this work and anonymous reviewers of this work for very useful comments that improved the exposition.

\section{References}

[ABSRW04] Michael Alekhnovich, Eli Ben-Sasson, Alexander A. Razborov, and Avi Wigderson. Pseudorandom generators in propositional proof complexity. SIAM J. Comput., 34(1):67-88 (electronic), 2004. 1

[AGHT20] Yaroslav Alekseev, Dima Grigoriev, Edward A. Hirsch, and Iddo Tzameret. Semi-algebraic proofs, IPS lower bounds, and the $\tau$-conjecture: can a natural number be negative? In 52nd Annual ACM SIGACT Symposium on Theory of Computing, STOC 2020, pages 54-67, 2020. $1,1.3,2.6$

[Ajt88] Miklós Ajtai. The complexity of the pigeonhole principle. In Proceedings of the IEEE 29th Annual Symposium on Foundations of Computer Science, pages 346-355, 1988. 1

[AM20] Albert Atserias and Moritz Müller. Automating resolution is NP-hard. J. ACM, 67(5):31:131:17, 2020. 1.1.2, 1.1.2, 1.3, 4.1, 4.7 
[BIK ${ }^{+}$96] Paul Beame, Russell Impagliazzo, Jan Krajíček, Toniann Pitassi, and Pavel Pudlák. Lower bounds on Hilbert's Nullstellensatz and propositional proofs. Proc. London Math. Soc. (3), 73(1):1-26, 1996. 2.2, 2.2

[CR74a] Stephen A. Cook and Robert A. Reckhow. Corrections for "On the lengths of proofs in the propositional calculus (preliminary version)". SIGACT News, 6(3):15-22, July 1974. 4.2

[CR74b] Stephen A. Cook and Robert A. Reckhow. On the lengths of proofs in the propositional calculus (preliminary version). In Proceedings of the 6th Annual ACM Symposium on Theory of Computing (STOC 1974), pages 135-148, 1974. For corrections see Cook-Reckhow [CR74a]. 4.2

[CR79] Stephen A. Cook and Robert A. Reckhow. The relative efficiency of propositional proof systems. J. Symb. Log., 44(1):36-50, 1979. This is a journal-version of Cook-Reckhow [CR74b] and Reckhow [Rec76]. 1

[Fri79] Harvey Friedman. On the consistency, completeness and correctness problems. Unpublished, 1979. 1.3

[FSTW16] Michael A. Forbes, Amir Shpilka, Iddo Tzameret, and Avi Wigderson. Proof complexity lower bounds from algebraic circuit complexity. In 31st Conference on Computational Complexity, CCC 2016, May 29 to June 1, 2016, Tokyo, Japan, pages 32:1-32:17, 2016. 1, 1.3, 2.2

[Gar19] Michal Garlík. Resolution lower bounds for refutation statements. In 44th Int. Symp. Math. Found. CS MFCS 2019, volume 138 of LIPIcs, pages 37:1-37:13, 2019. 1.1.2, 1.1.2, $4.1,4.7$

[GKRS19] Mika Göös, Pritish Kamath, Robert Robere, and Dmitry Sokolov. Adventures in monotone complexity and TFNP. In 10th Innovations in Theoretical Computer Science Conference, ITCS 2019, January 10-12, 2019, San Diego, California, USA, pages 38:1-38:19, 2019. 1

[GP18] Joshua A. Grochow and Toniann Pitassi. Circuit complexity, proof complexity, and polynomial identity testing: The ideal proof system. J. ACM, 65(6):37:1-37:59, 2018. 1, 1.1.1, 1.2, 1.3, 2.2, $2.2,2.4,3.1 .1,3.1 .1,3.2,3.2,3.2 .1$

[Hak85] Armin Haken. The intractability of resolution. Theoret. Comput. Sci., 39(2-3):297-308, 1985. 1

[KPW95] Jan Krajíček, Pavel Pudlák, and Alan Woods. An exponential lower bound to the size of bounded depth Frege proofs of the pigeonhole principle. Random Structures Algorithms, $7(1): 15-39,1995.1$

[Kra95] Jan Krajíček. Bounded arithmetic, propositional logic, and complexity theory, volume 60 of Encyclopedia of Mathematics and its Applications. Cambridge University Press, Cambridge, 1995. 13

[Kra97] Jan Krajíček. Interpolation theorems, lower bounds for proof systems, and independence results for bounded arithmetic. The Journal of Symbolic Logic, 62(2):457-486, 1997. 1

[Kra01] Jan Krajíček. Tautologies from pseudo-random generators. Bull. Symbolic Logic, 7(2):197-212, 2001. 1

[Kra04a] Jan Krajíček. Diagonalization in proof complexity. Fundamenta Mathematicae, 182:181-192, 2004. 1.3

[Kra04b] Jan Krajíček. Dual weak pigeonhole principles, pseudo-surjective functions, and provability of circuit lower bounds. Journal of Symbolic Logic, 69(1):265-286, 2004. 1.1.1

[Kra04c] Jan Krajíček. Implicit proofs. Journal of Symbolic Logic, 69(2):387-397, 2004. 1.3

[Kra11] Jan Krajíček. On the proof complexity of the Nisan-Wigderson generator based on a hard NP $\cap$ coNP function. J. Math. Log., 11(1):11-27, 2011. 3 
[Kra19] Jan Krajíček. Proof complexity, volume 170. Cambridge University Press, 2019. 4.1

[LTW18] Fu Li, Iddo Tzameret, and Zhengyu Wang. Characterizing propositional proofs as noncommutative formulas. In SIAM Journal on Computing, volume 47, pages 1424-1462, 2018. Full Version: http://arxiv.org/abs/1412.8746. 1, 1.3

[PBI93] Toniann Pitassi, Paul Beame, and Russell Impagliazzo. Exponential lower bounds for the pigeonhole principle. Comput. Complexity, 3(2):97-140, 1993. 1

[PS19] Jan Pich and Rahul Santhanam. Why are proof complexity lower bounds hard? In 60th Annual IEEE Symposium on Foundations of Computer Science FOCS 2019, November 9-12, 2019, Baltimore, Maryland USA, 2019. 1, 1.1.2, 1.3, 4.9, 4.2, 4.2

[PT16] Tonnian Pitassi and Iddo Tzameret. Algebraic proof complexity: Progress, frontiers and challenges. ACM SIGLOG News, 3(3), 2016. 1

[Pud86] Pavel Pudlak. On the length of proofs of finitistic consistency statements in first order theories. Studies in Logic and the Foundations of Mathematics, 120:165-196, 1986. 1.3

[Pud87] Pavel Pudlak. Improved bounds to the length of proofs of finite consistency statements. Contemporary Mathematics, 65:309-331, 1987. 1.3

[Pud20] Pavel Pudlak. Reflection principles, propositional proof systems, and theories. ArXiV, July 2020. 4, 1.3, 4.1

[Raz10] Ran Raz. Elusive functions and lower bounds for arithmetic circuits. Theory of Computing, 6(1):135-177, 2010. 1.2, 3.1.2, 3.9

[Raz15] Alexander A. Razborov. Pseudorandom generators hard for $k$-DNF resolution and polynomial calculus resolution. Annals of Mathematics, 181:415-472, 2015. 1, 1.1.1, 3

[Raz16] Alexander Razborov. Propositional proof complexity: Fifteen (or so) years after. Talk at "A Celebration of Mathematics and Computer Science. Celebrating Avi Wigderson's 60th Birthday October 5 - 8, 2016". https://youtu. be/7LfW6VTW8zo?t=2722, 2016. 3

[Raz21] Alexander Razborov. P, NP and proof complexity. Talk at "SAT and Foundations of Mathematics", Simons Institute. https://youtu.be/xx4mxcqA15A?t=2333, 2021. 3

[Rec76] Robert A. Reckhow. On the lengths of proofs in the propositional calculus. PhD thesis, University of Toronto, 1976. 4.2

[RR97] Alexander A. Razborov and Steven Rudich. Natural proofs. J. Comput. System Sci., 55(1, part 1):24-35, 1997. Proceedings of the 26th Annual ACM Symposium on the Theory of Computing (STOC '94) (Montreal, PQ, 1994). 1.3

[Rud97] Steven Rudich. Super-bits, demi-bits, and NP/qpoly-natural proofs. In RANDOM'97, Proceedings, volume 1269, pages 85-93, 1997. 1.1.2, 4.10

[SS95] Michael Shub and Steve Smale. On the intractability of Hilbert's Nullstellensatz and an alge-

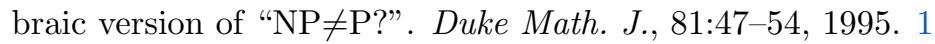

[Str73] Volker Strassen. Vermeidung von divisionen. J. Reine Angew. Math., 264:182-202, 1973. (in German). 3.1.2, 2

[SY10] Amir Shpilka and Amir Yehudayoff. Arithmetic circuits: A survey of recent results and open questions. Foundations and Trends in Theoretical Computer Science, 5(3-4):207-388, 2010. 2.1, 2.1, 3.1 .2

[Val79a] Leslie G. Valiant. Completeness classes in algebra. In Proceedings of the 11th Annual ACM Symposium on the Theory of Computing, pages 249-261. ACM, 1979. 2.1, 3.1.3

[Val79b] Leslie G. Valiant. The complexity of computing the permanent. Theor. Comput. Sci., 8:189-201, 1979. 2.1, 3.2.1

[Val82] Leslie G. Valiant. Reducibility by algebraic projections. Logic and Algorithmic: International Symposium in honour of Ernst Specker, 30:365-380, 1982. 2.1 
— Page left blank for ECCC stamp — 